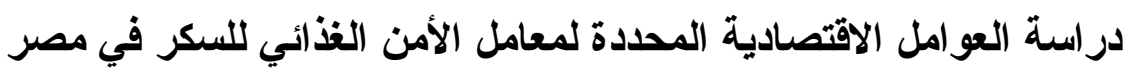
ألاء جمال إسماعيل، محمد عبد الوهاب أبونحول، عاطف حلمى الثيمى وطلعت حافظ إسماعيل

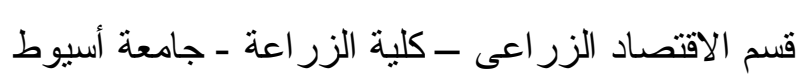

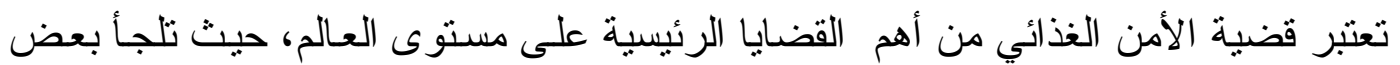

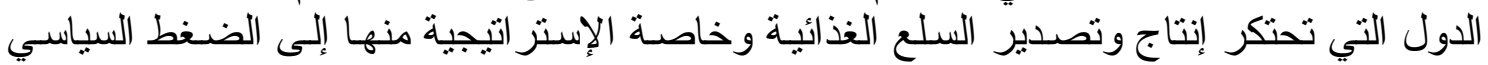

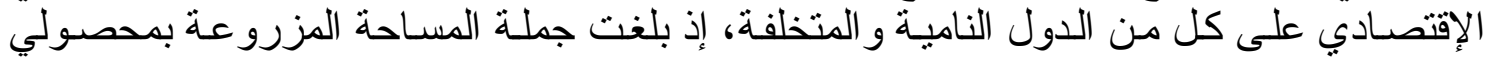

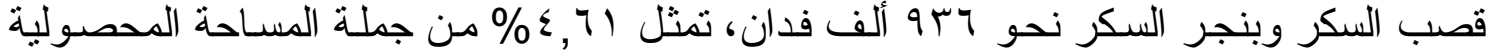

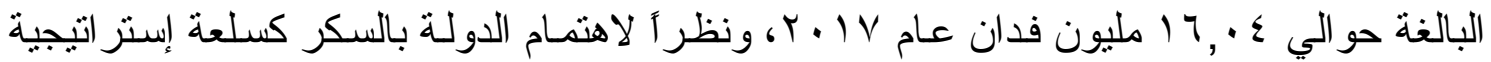

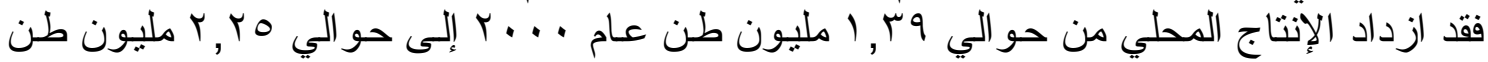

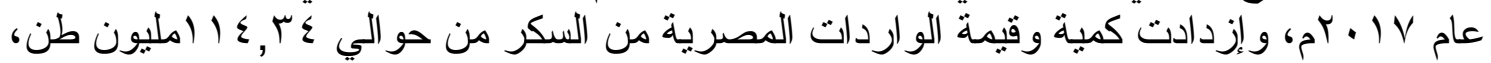

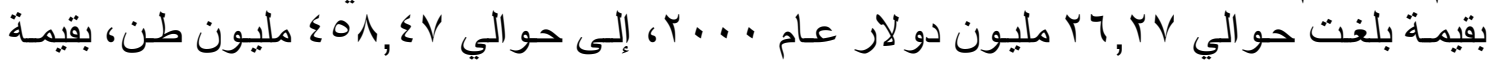

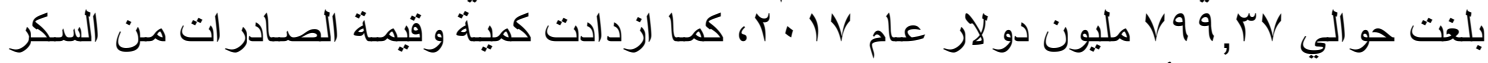

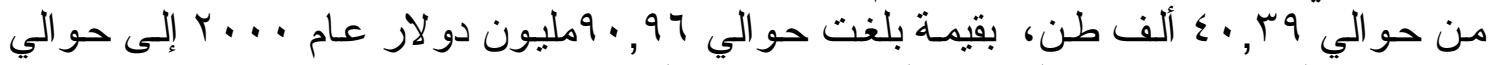

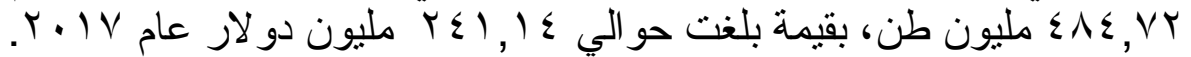

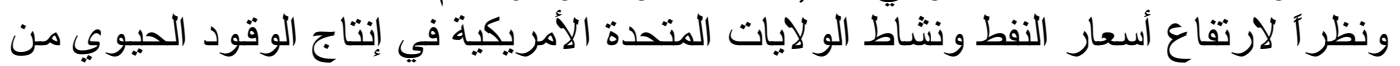

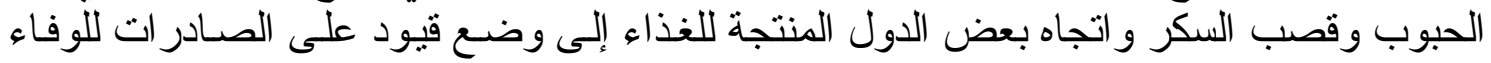

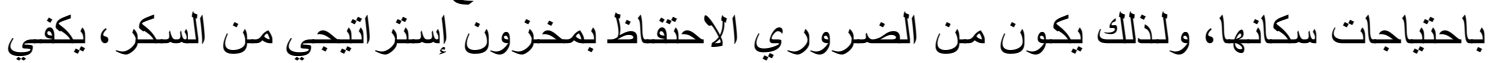

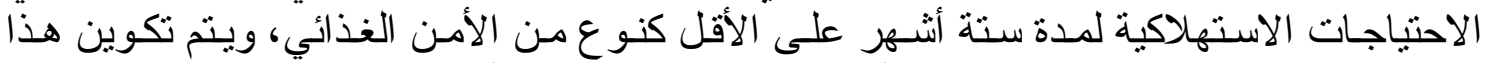

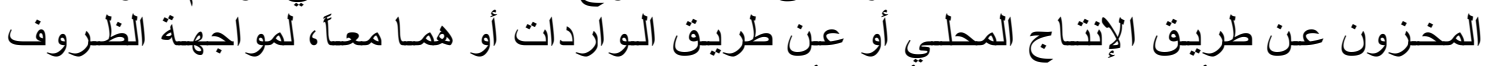
الطارئة، خاصة و أن منطقة الثرق الأوسط أصبحت غير مستقرة سياسيأ و اقتصاديا.

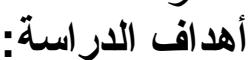

استهدفت هذه الدر اسة التعرف على البعد الاقتصادي لسياسة الأمن الغذائي للسكر في مصر وذللك من خلال تحقيق الأهداف الفر عية التالية:

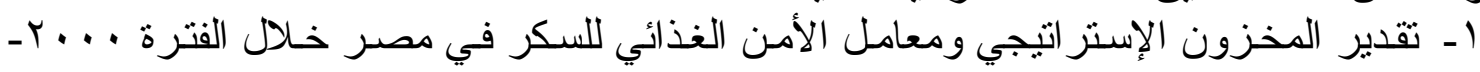
$r+1 V$ r- در اسـة العو امـل الاقتصـادية المحـددة لمعامـل الأمـن الغذائي للسكر في مصـر خـلال فتـرة الدر اسة.

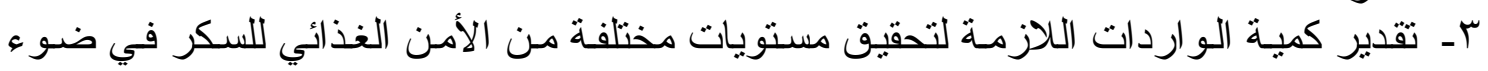

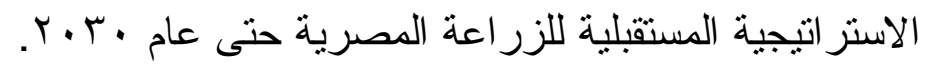

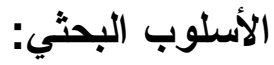
اعتمدت هذه الدر اسة في تحقيق أهدافها على التحليل الاقتصادي الكمي، و على وجه التحديد نم استخدام المعادلات و النماذج التالية:

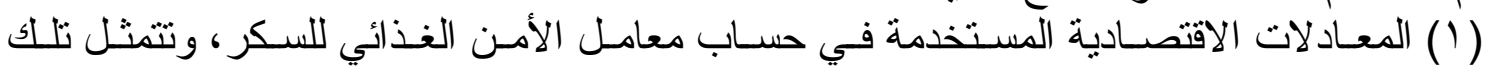

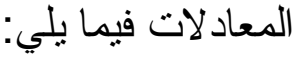

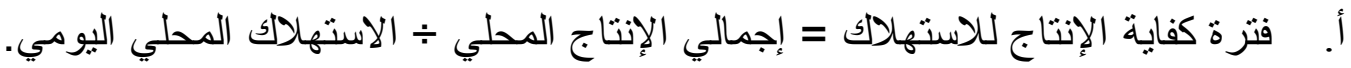

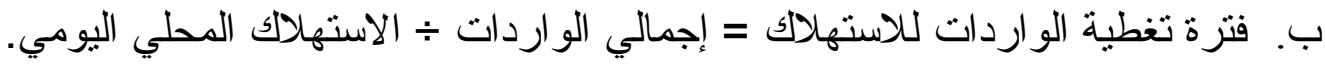

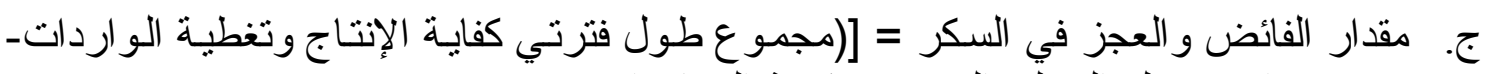

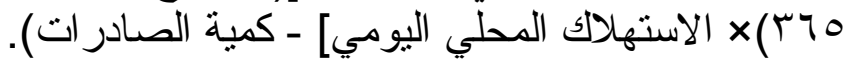


د. معامل الأمسن الغذائي للسكر = حجم المخزون الإستر اتيجي ( محصلة الفائض و العجز) ) متوسط الاستهلاك المحلي السنوي.

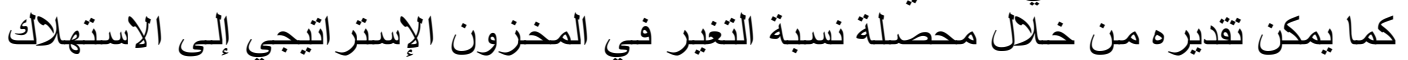

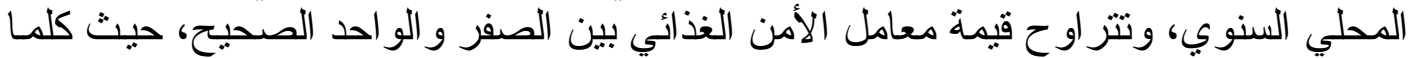

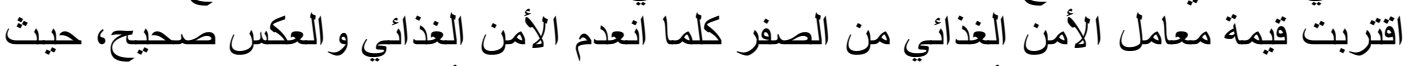

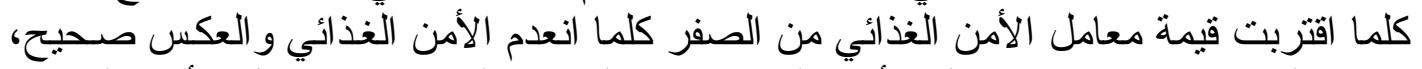

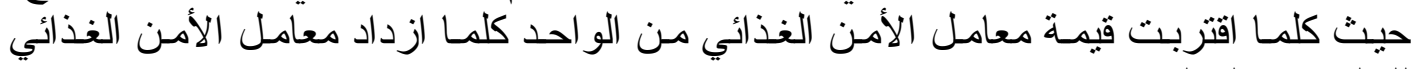

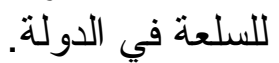

(r) تحليل الاتحدار المتعدد Multi Regression Analysis في در اسـة أثر العو امل الاقتصادية

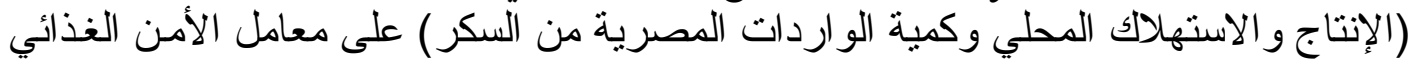

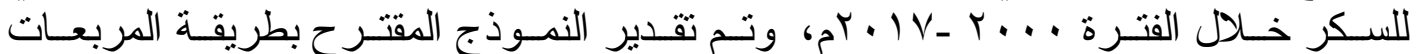

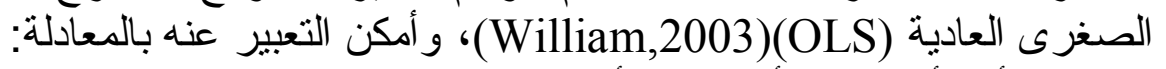

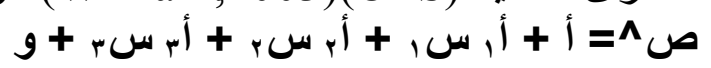

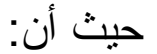
ص ^ تمثل معامل الأمن الغذائي للسكر في مصر.

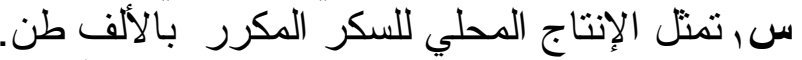

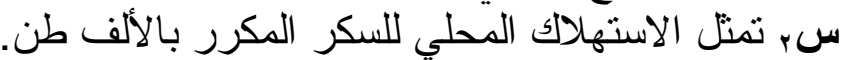

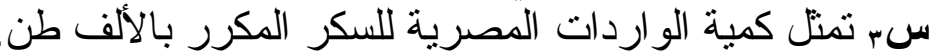

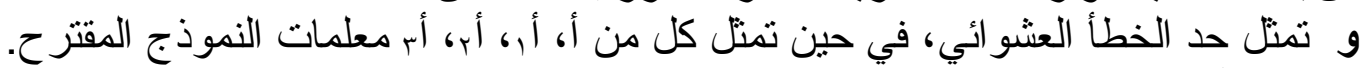

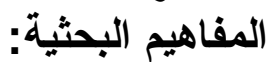
1- الأمن الغذائية: Food Security:

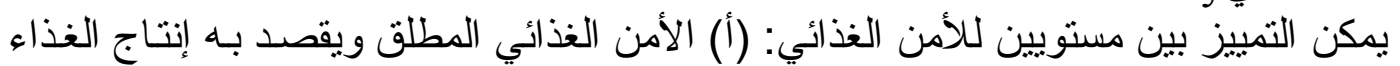

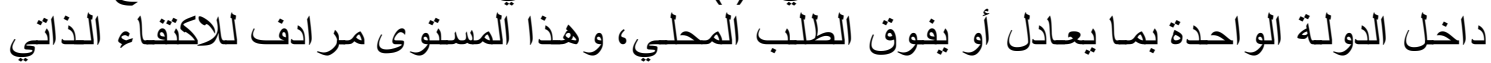

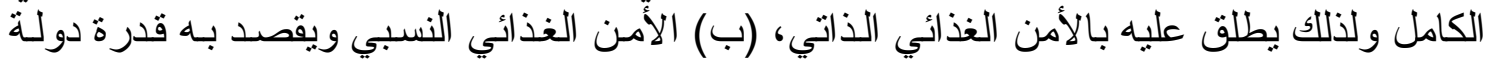

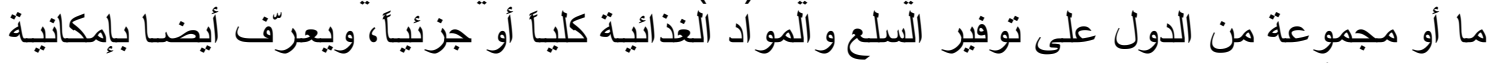

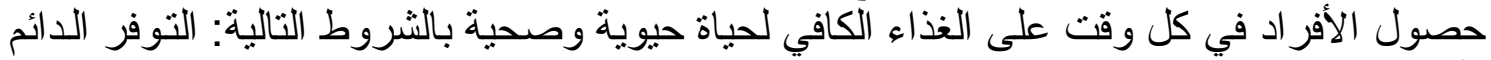

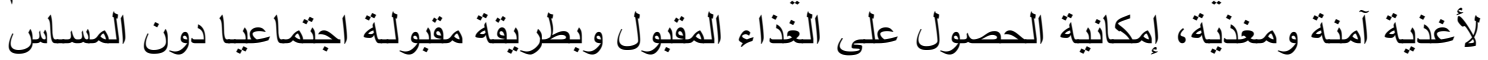

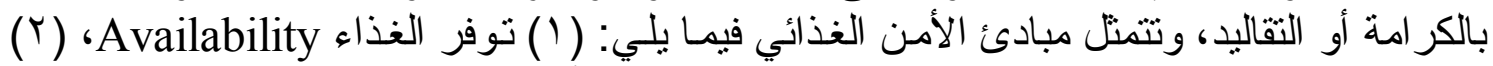

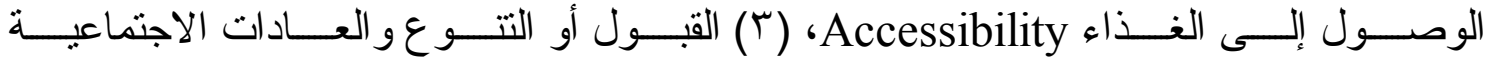
Agency Acceptability

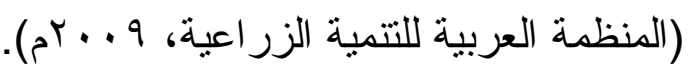
r- المخزون الإستر اتيجي Strategic Stock

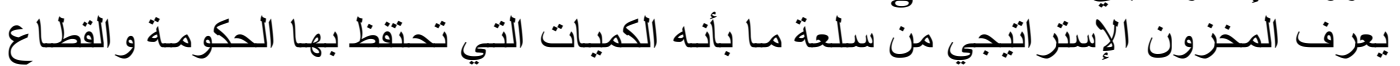

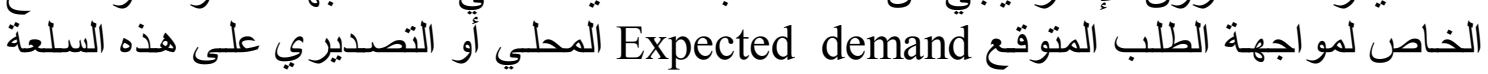

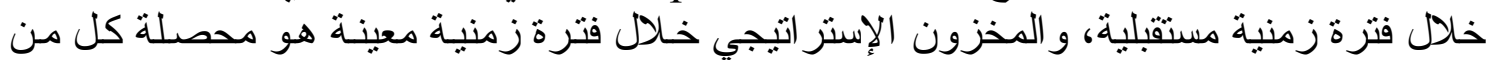

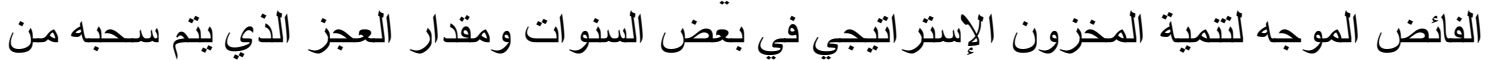

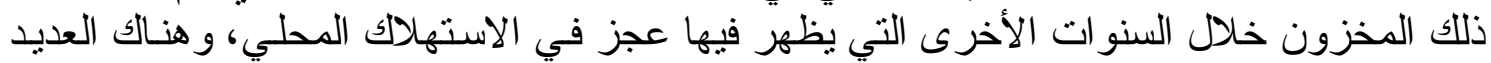

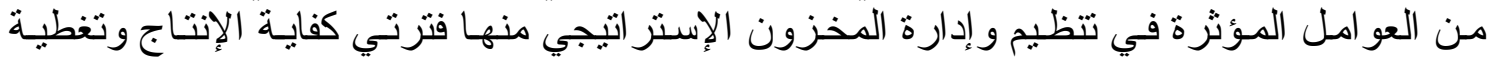

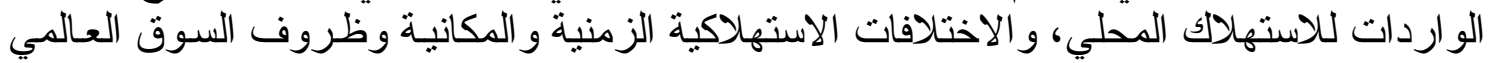

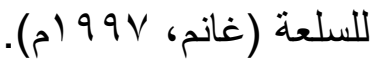


نتائج الار اسة:

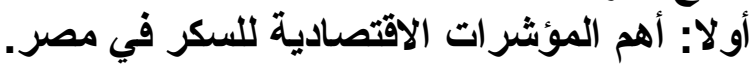
يتتاول هذا الجز ء من الدر استة بالعرض واليكر في ولتحليل أهم المؤشر ات الاقتصادية للمحاصيل السكرية وهي: تعداد السكان، كمية الواردات، كمية الاستهلاك، حجم الفجوة، نصيب الفئه الفرد في السنة، استهلاكك الفرد في السنة، نسبة الاكتفاء الذاتي، سعر التجزئة الأنة، سعر السكر عالمياً. ا ـ تطوز كمية الواردات من السكرئ

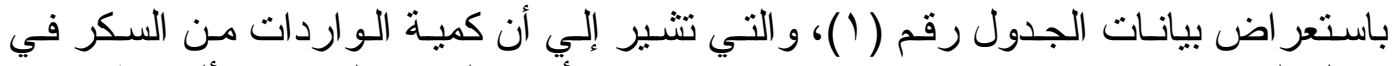

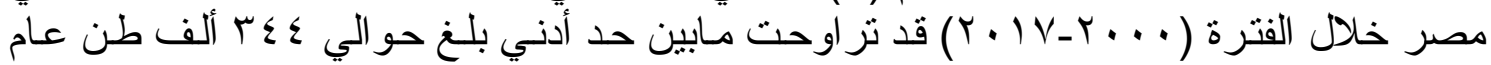

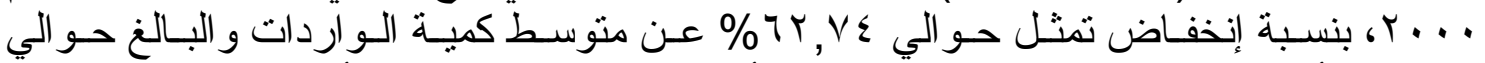

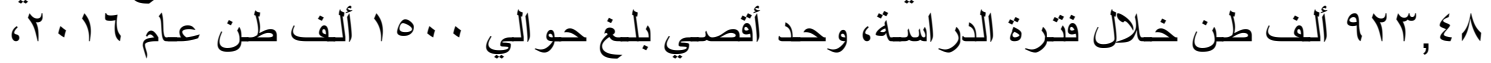

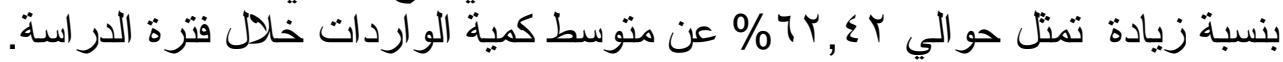

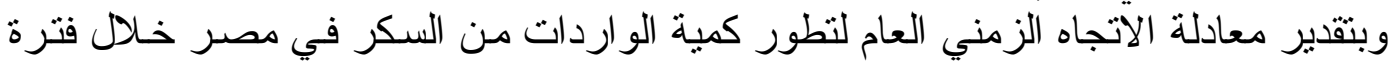

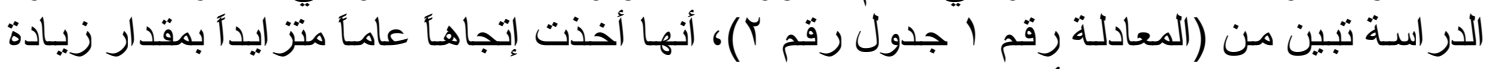

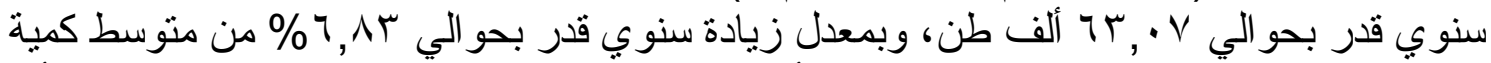

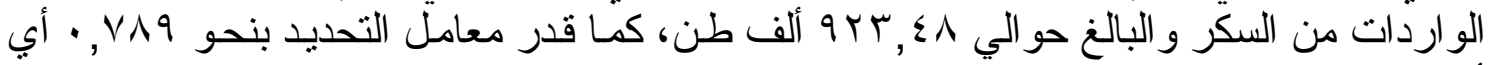

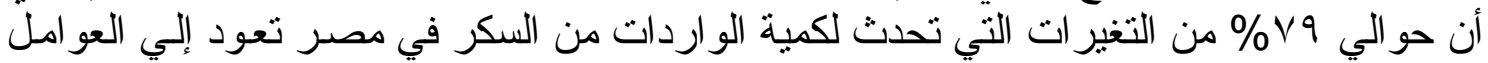

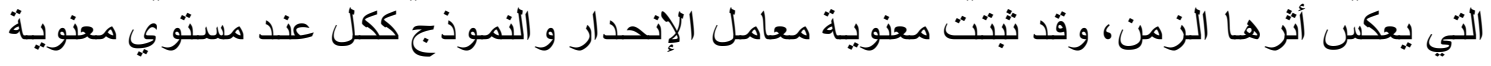
$\therefore$ (1) r بـ تطور الكمية المستهلكة من السكر:

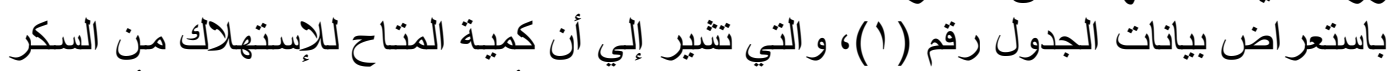

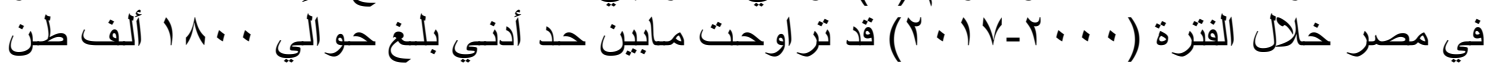

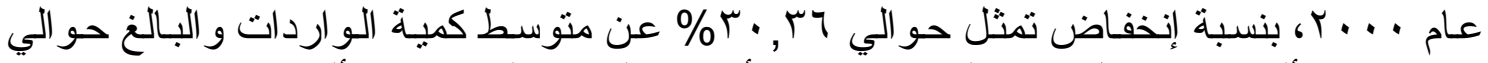

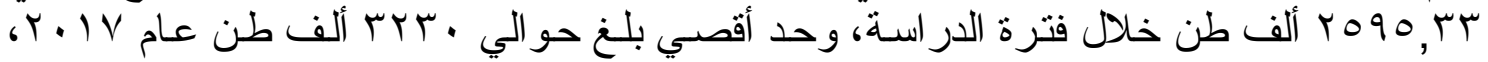

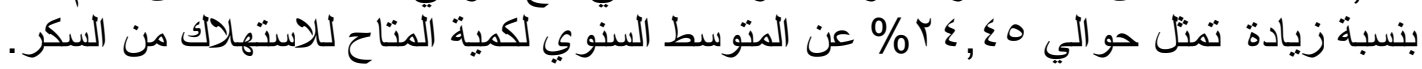

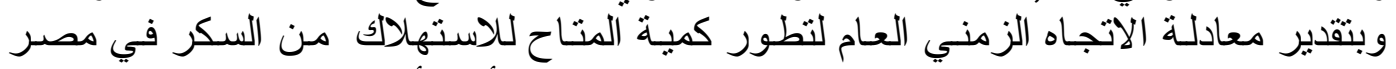

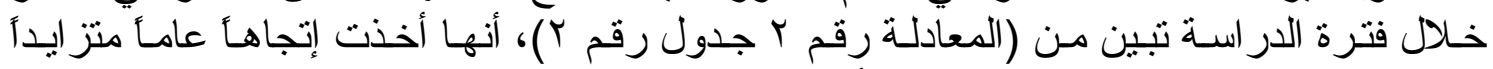

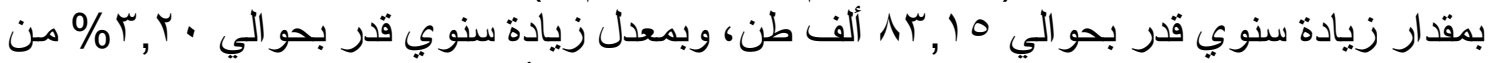

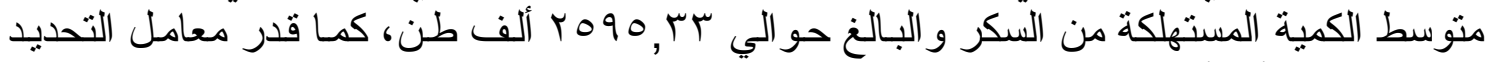

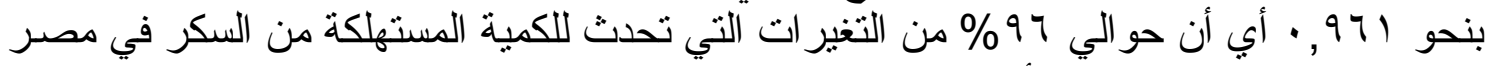

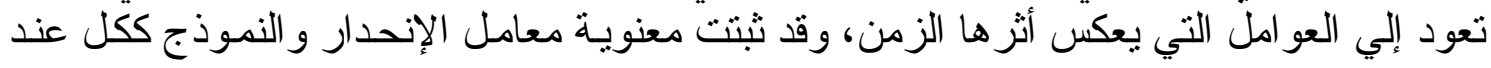

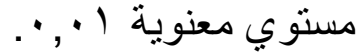




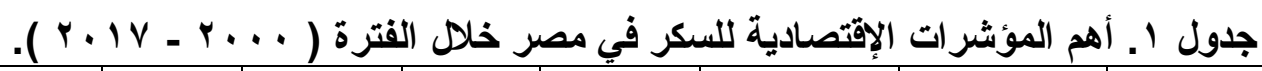

\begin{tabular}{|c|c|c|c|c|c|c|c|c|}
\hline مليون نسداد السكان & دولالار/طيً & جنية/كزئة & الألتي أنفاء & كجم/سنب & ألف طجمث & ألمستهنكة & كمية الوارد ألف طن & السنة \\
\hline $7 \varepsilon, 11$. & $r \ldots$ & $r, 0$. & $V V, \Sigma 1$ & $r V, 0$ & $\varepsilon \cdot V$ & $1 \wedge \ldots$ & $r \leqslant T, T \varepsilon$ & $r \ldots$ \\
\hline $70, r \ldots$ & 180 & $r, 0$. & $V \vee, \wedge O$ & $r V, \cdot$ & $\varepsilon \cdot 1$ & 11.7 & r9o,.. & $r \ldots 1$ \\
\hline $7 \vee, 9 \vee$. & $1 \leq .1$ & $r, \cdots$ & 71,74 & $r q, r$ & $7 \wedge r$ & $r \ldots$ & $0 . r, 7 \pi$ & $r \cdot r$ \\
\hline $79, r \ldots$ & ITA & $r, \cdots$ & $71, Y$. & $r \cdot r$ & 110 & YI.. & roß,AT & $r \cdot r r$ \\
\hline$V \cdot, 7 V \cdot$ & IVT & $r, 0$. & $7 Y, Y O$ & $r, 1$ & AM & YY.. & $\wedge r \tau, O \wedge$ & $Y \ldots \varepsilon$ \\
\hline$V Y, \cdot 1 \cdot$ & rrA & $r, 0$. & 71,09 & ru, & 940 & $r \leq r r$ & $\pi \cdot, \wedge 1$ & $r \ldots o$ \\
\hline$V \Gamma, \tau \varepsilon$. & $r .0$ & $r, \cdots$ & $71, V 1$ & $r \varepsilon$, & $9 \vee \wedge$ & roor & $q \vee 0, r V$ & $Y . T$ \\
\hline $\mathrm{Vo,19.}$ & TIT & $r, \cdots$ & $7 V, 71$ & $r \varepsilon, \cdot$ & 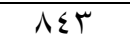 & Y7.. & $\vee \vee \cdot, \wedge l$ & $r \cdot . V$ \\
\hline$V 7,9 \mu$. & rys & r,Vo & $09,9 \leq$ & $r \varepsilon, \cdot$ & 1.01 & YTE. & $1171, \leqslant 9$ & $r \ldots \lambda$ \\
\hline$\vee \wedge, 79$. & $\varepsilon T$ & $r, r v$ & $09, Y Y$ & $r \varepsilon, \cdot$ & 111. & TVY. & 701,19 & $r .99$ \\
\hline 1.,Or. & $\varepsilon \vee 9$ & $\varepsilon, \Sigma \Gamma$ & $V Y, \cdot Y$ & $\Gamma \varepsilon$, & V79 & YVTO & $1 \cdot v \leqslant, 0 \leqslant$ & $r \cdot 1 \cdot$ \\
\hline$\Delta r, 00$. & OVO & $0,0 \leqslant$ & $79, \cdot 1$ & $\Gamma \varepsilon$, & Nor & TVO. & $11 \leqslant \varepsilon, r_{0}$ & $r .11$ \\
\hline 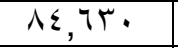 & $\leqslant 00$ & $0, V \varepsilon$ & $v \cdot, \cdot \lambda$ & $r \varepsilon, \cdot$ & 107 & YAT. & $1.0 ., r r$ & $r .1 Y$ \\
\hline$\wedge \neg, \wedge)$. & rvq & $0, \leqslant Y$ & 77,09 & $r \varepsilon, \cdot$ & $\ldots r$ & $r \ldots$ & $1 \Gamma \cdot \varepsilon, \cdots$ & $r .1 r$ \\
\hline$\wedge \wedge, 97$. & r. & 0,19 & $V 7,71$ & $r \varepsilon, \cdot$ & VYI & $\Gamma \ldots$ & IrA.,.. & $Y \cdot 1 \leq$ \\
\hline $91, \cdot r$. & TAY & $0, \wedge \wedge$ & $v 7$, or & $r \varepsilon, \cdot$ & VYA & W... & $1 \leq 7, \ldots$ & $r .10$ \\
\hline$q r, 1 r$. & 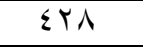 & $\Lambda, r$ & $79,0 Y$ & $r \varepsilon, \cdot$ & $97 \varepsilon$ & T17. & $10 \ldots, \ldots$ & $Y .17$ \\
\hline $90, \ldots$ & r99 & $1 \varepsilon, \cdot \varepsilon$ & 79,71 & $r \varepsilon$, & 911 & TrT. & $1 \cdot V T, 0$. & $Y \cdot I V$ \\
\hline$\vee \wedge,\rceil \varepsilon$ & 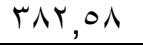 & $\varepsilon, 01$ & $7 V, 94$ & Tr,oT & $\wedge Y q, \vee r$ & ro90,rr & $q Y T, \leqslant \wedge$ & المتوسط \\
\hline
\end{tabular}

(1) وز ارة الزر اعة و استصلاح الأر اضي، قطاع الثئون الاقتصـادية، الإدارة المركزيـة للإقتصـاد الزر اعي، نشرة الإحصـاءات

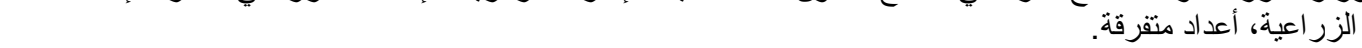

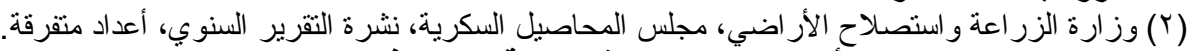

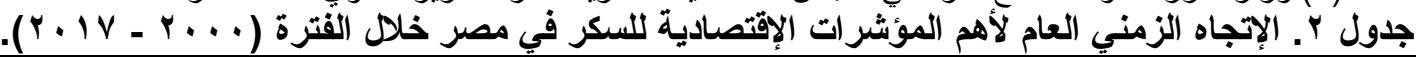

\begin{tabular}{|c|c|c|c|c|c|c|c|}
\hline التغير \% & 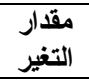 & المتوسط & 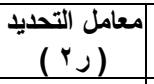 & قالمسيوة (ف) & المعـــــــادلة & الظاهرة & المعادلة \\
\hline$\urcorner, \wedge r$ & $\pi, \cdot v$ & $9 r \pi, \leqslant 1$ & $\cdot, \vee \wedge 9$ & **०१, Vr & 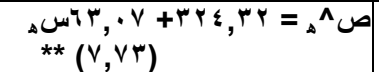 & 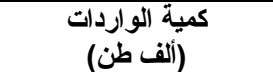 & (1) \\
\hline$r, r$. & NT, 10 & ro90,rr & $\cdot, 971$ & **ץ११, ह1 & 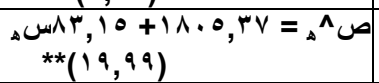 & الكمية المستهلكة & (Y) \\
\hline$r, r r$ & $11,0 \leqslant$ & $\Delta r q, V r$ & $\cdot$, YON & $*_{0}, 00$ & 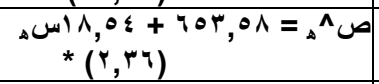 & حجم الفجوة & $(r)$ \\
\hline 1,11 & אדצי, & rT, OT & ט גזT, & $* * Y \wedge, r)$ & 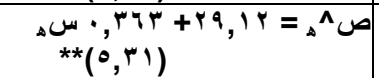 & نصيب الفرد كجم/سنة & $(\varepsilon)$ \\
\hline- & - & $T V, q T$ & $\cdot, \cdot r_{1}$ & דזr, & 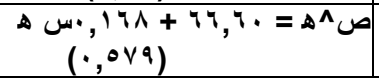 & نسبة الاكتفاء الذاتي \% & $(0)$ \\
\hline $1 \cdot, 11$ & $\cdot, \leqslant 07$ & $\{, 01$ & $\cdot, T V T$ & 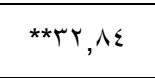 & 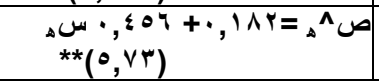 & سعر التجزئة جنية/كجم & (7) \\
\hline- & - & rAr,ON & $\cdot, \cdots 1$ & $\cdot, \cdots \varepsilon$ & 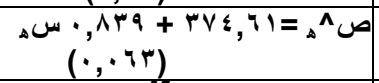 & سعر السكر عالميا & (V) \\
\hline$r, r$ & - & $\vee \wedge, T \leqslant$ & $\cdot, 999$ & $* * \|$ । $q$ r, $q$ & 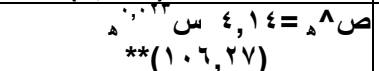 & تعداد السكان مليون نسمة & $(\wedge)$ \\
\hline
\end{tabular}

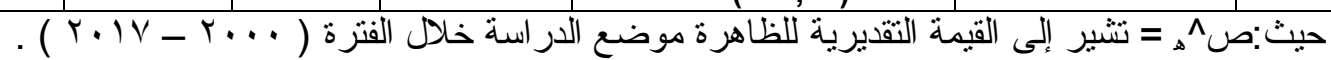

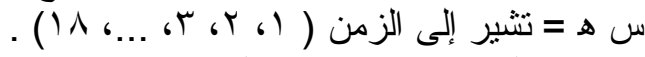

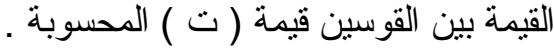

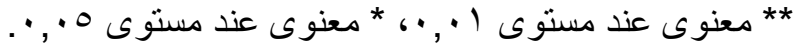

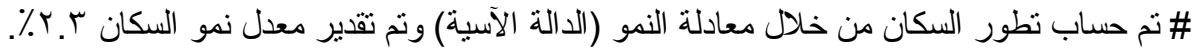
المصدر : جمعت وحسبت من: بيانات جدول رقم (1) (1). 


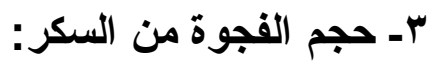

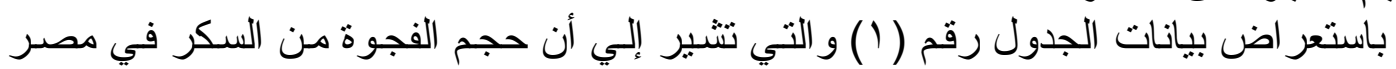

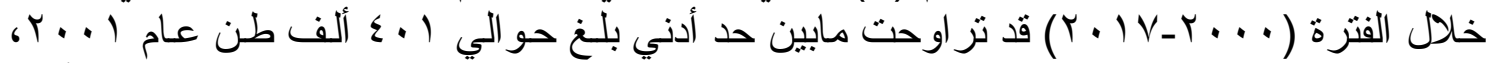

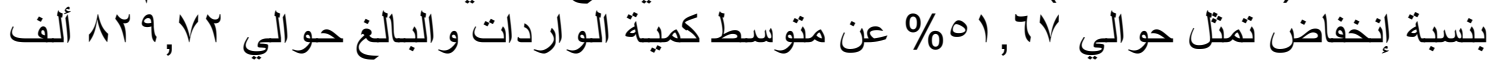

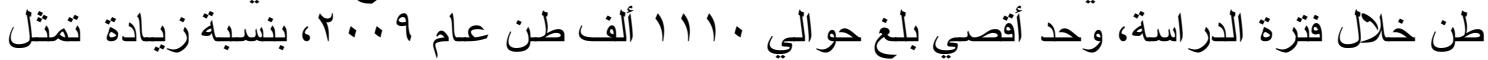

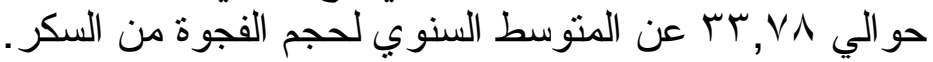

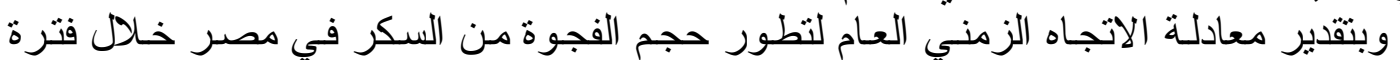

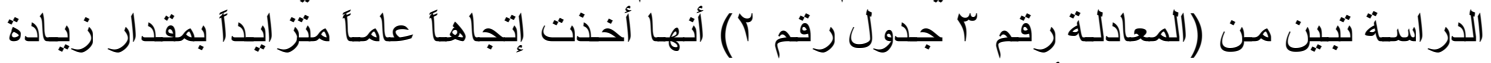

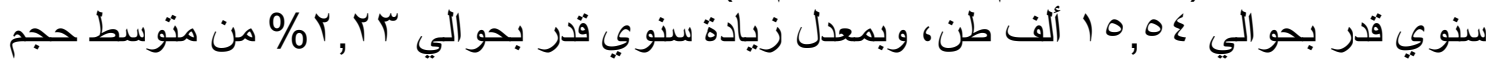

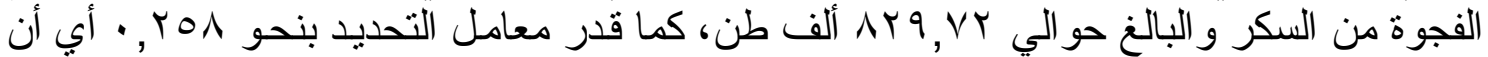

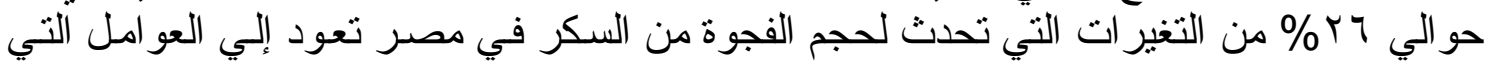

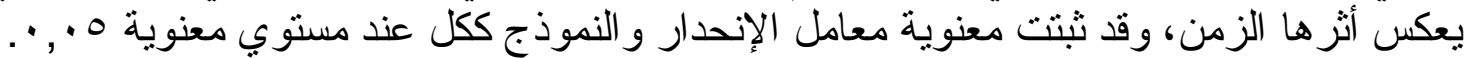

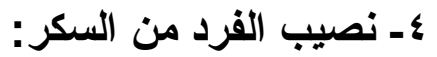

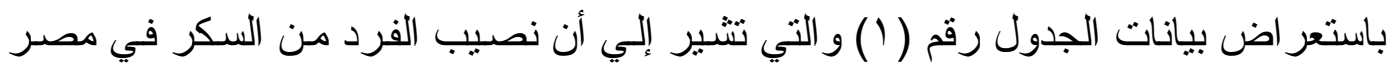

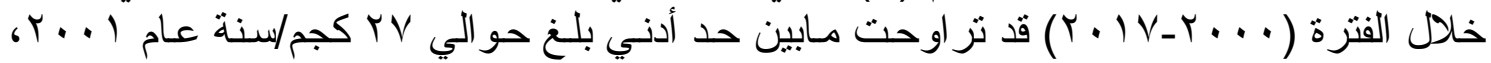

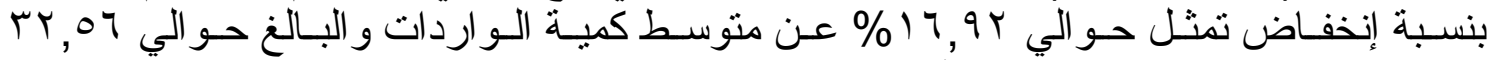

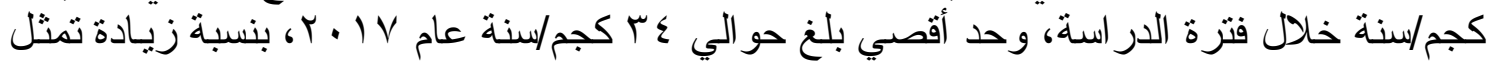

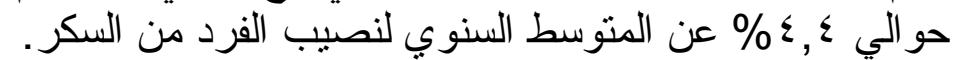

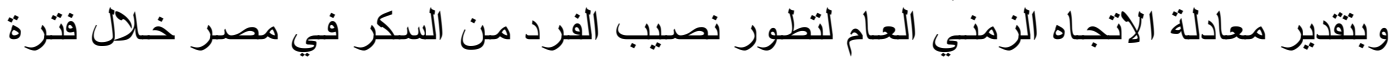

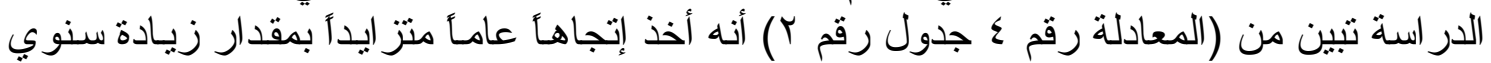

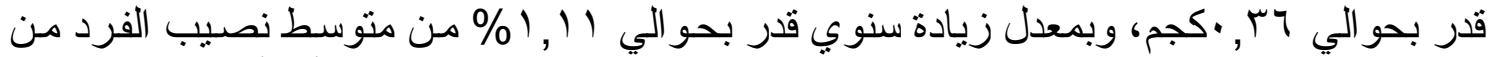

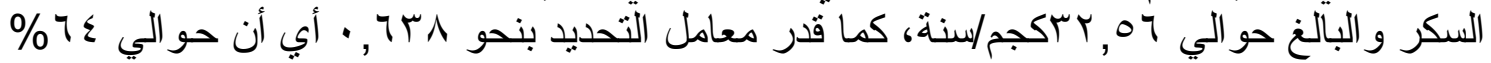

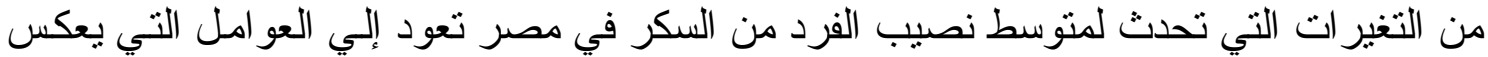

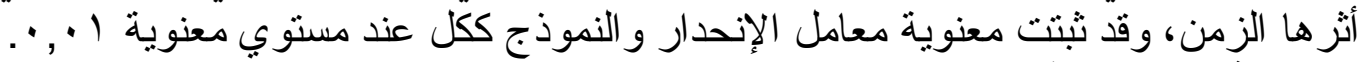
هـ نسبة الإكتفاء الأتي من فن السكر:

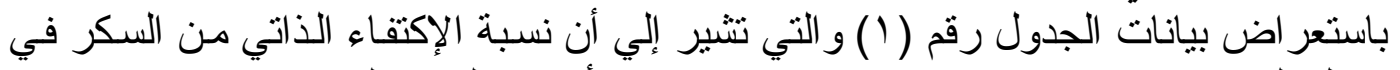

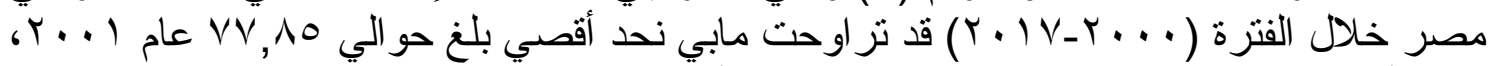

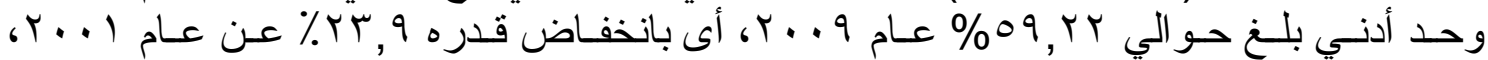

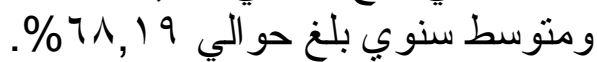

وبتقدير معادلة الاتجاه الزمني العام لتطور نسبة الإكتقاء الذاتي من السكر في مصر خـلال

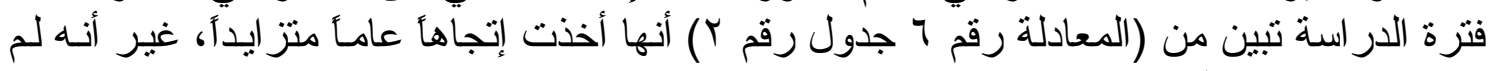
تثتبت معنوية هذه الزيادة.

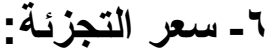

باستعر اض بيانات الجدول رقم ( (1) و التي تشير إلبي أن سعر التجزئة في مصر خـالال الفترة

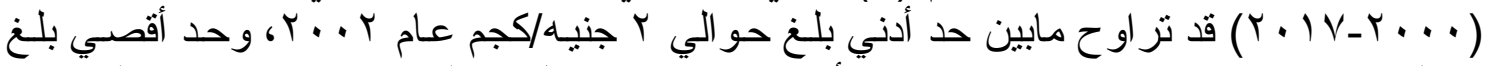

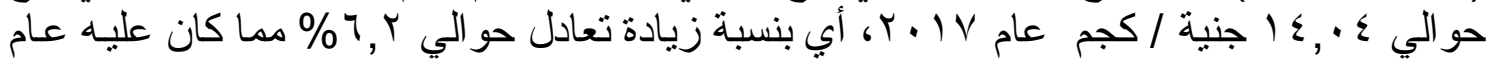

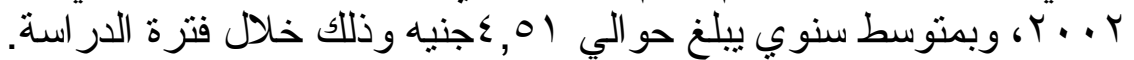

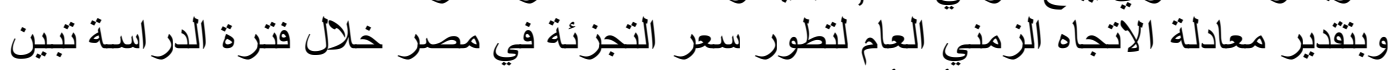

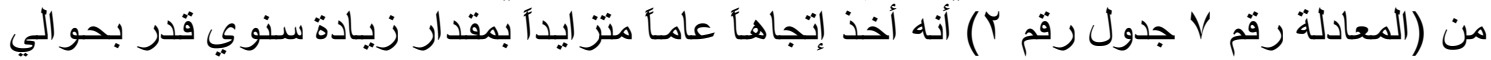

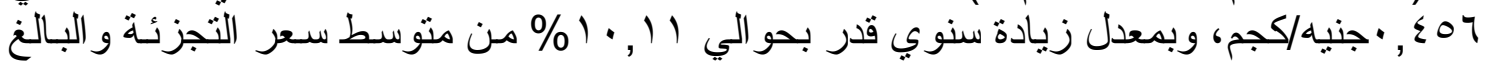

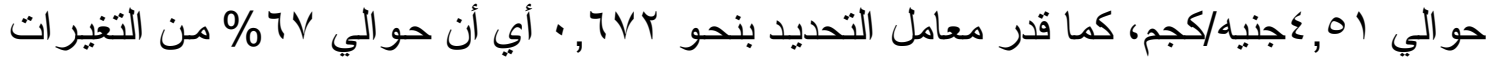


التي تحدث لسعر التجزئة في مصر تعود إلي العو امل التي يعكس أثزهـا الزمن، وقد ثبتـت معنويـة

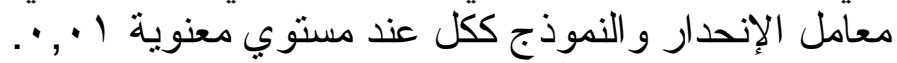

V- سعر السكر عالمياً:

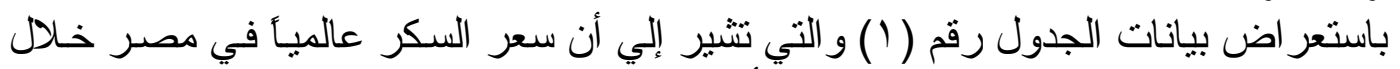

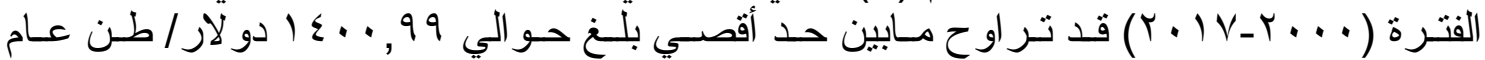

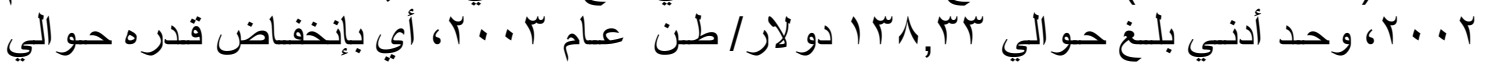

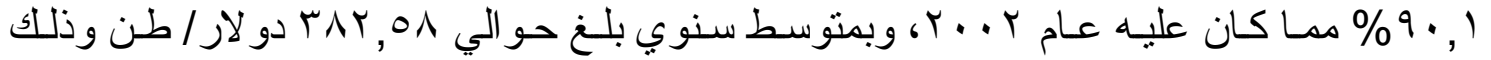
خلال فترة الدر اسة.

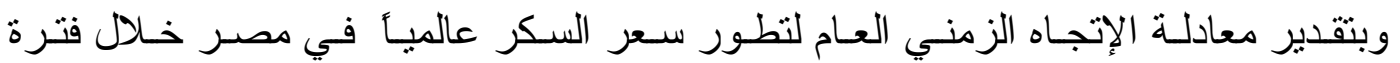

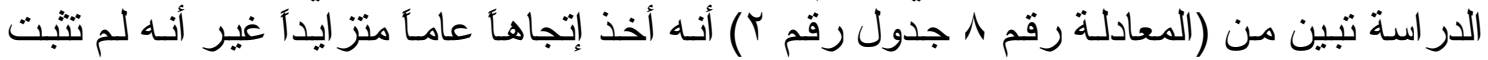
معنوية هذه الزيادة.

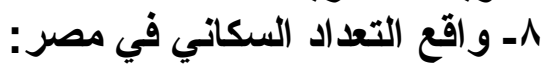

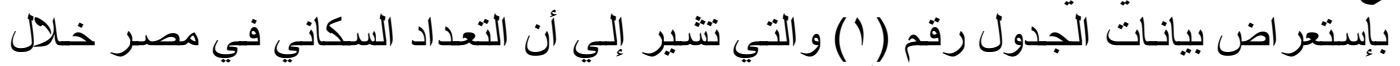

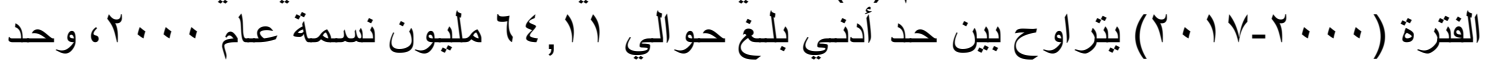

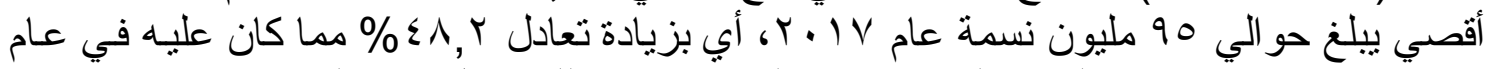

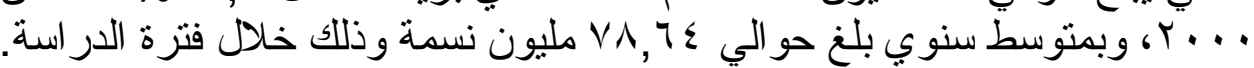

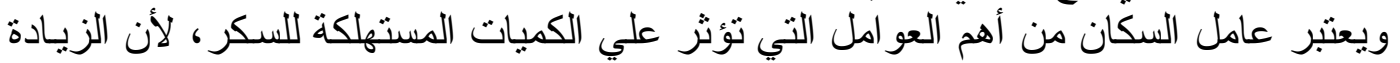

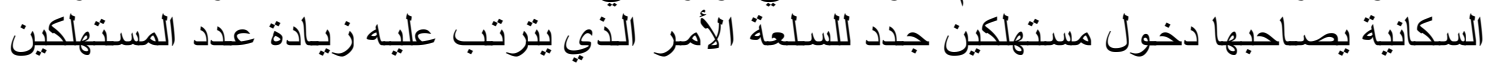

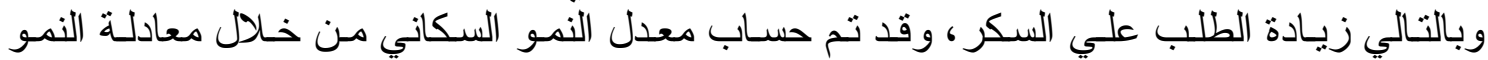

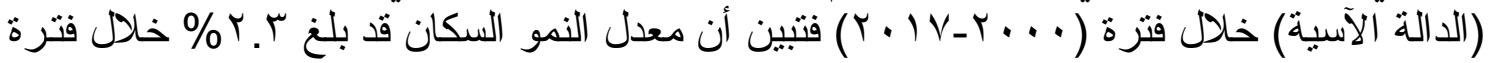

ثانياً: تقدير المخزون الاستراتيجي ومعامل الأمن الغذائي للسكر في مصر.

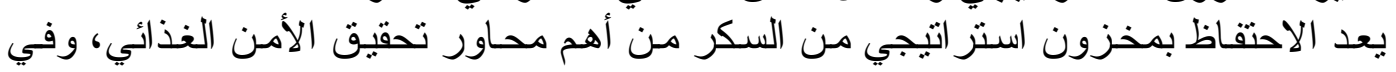

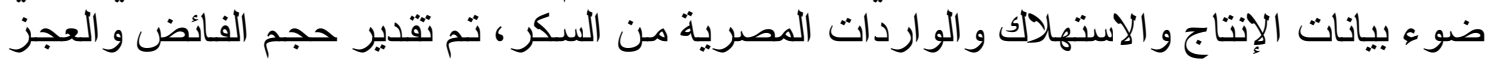

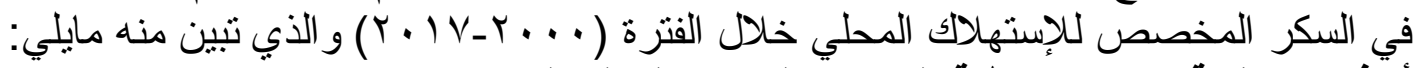

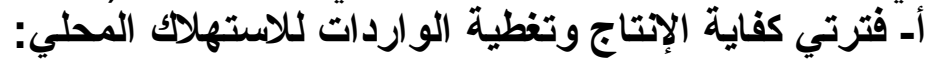

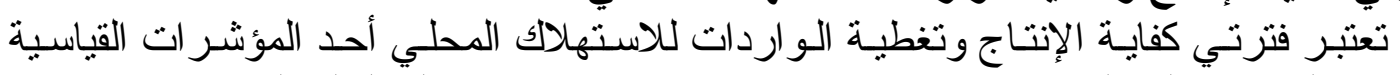

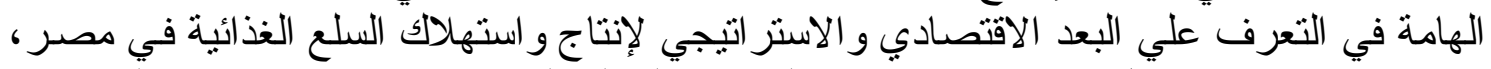

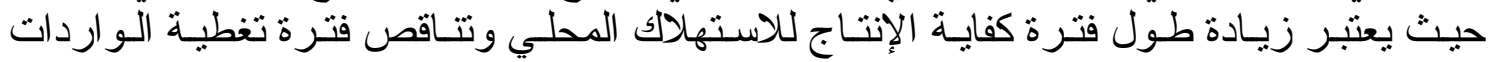

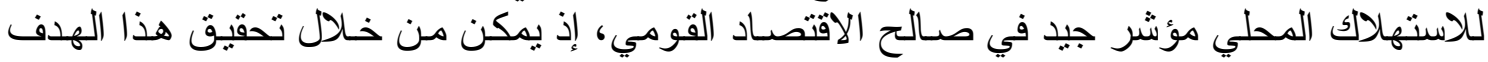

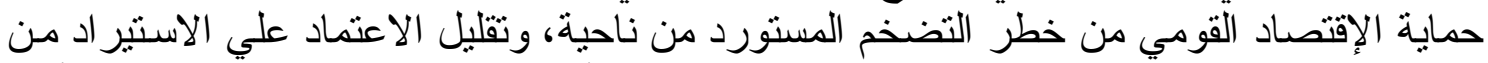

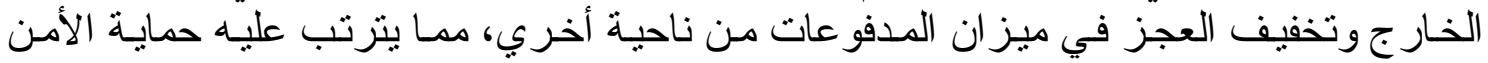

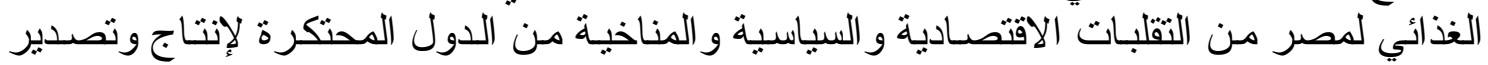

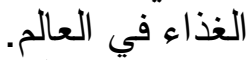

\section{ا ـ فترة كفاية الإنتاج للاستهلافك المحلي:}

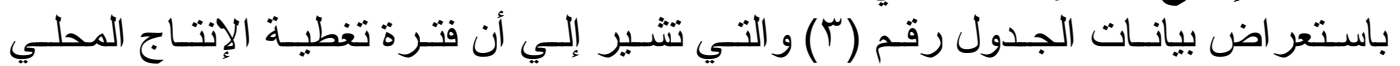

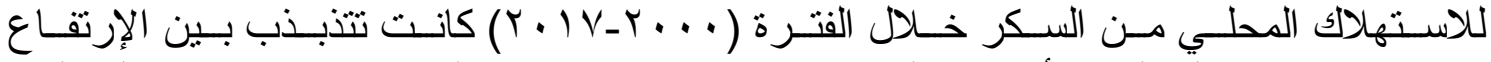

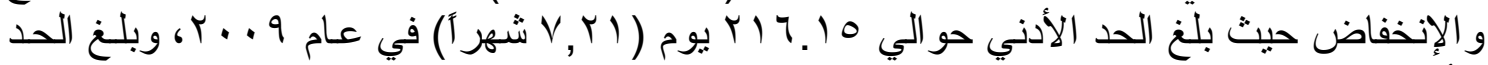

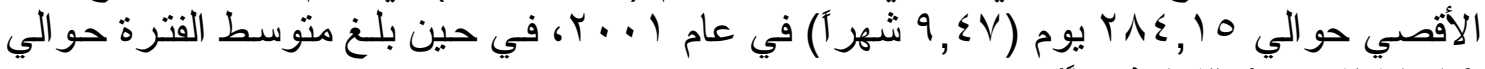
(َ). 
r- فترة تغطية الواردات للإستهلاك المحلي:

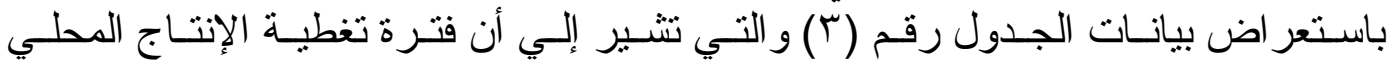

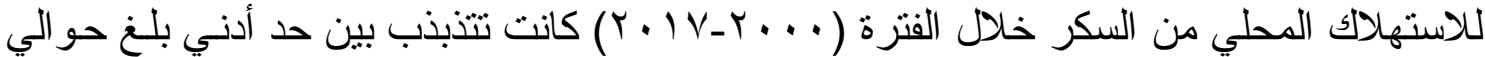

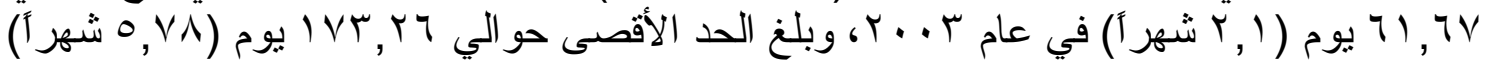

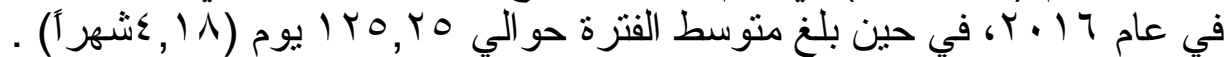
ب- المخزون الاستّر اتيجي ومعامل الأمن الغذائي:

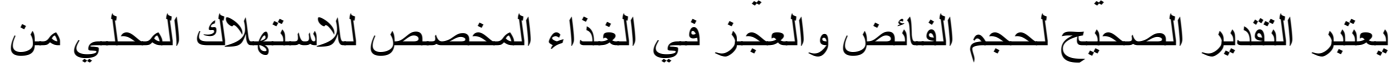

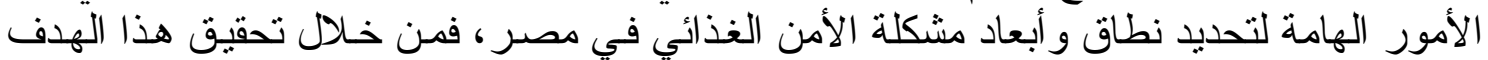

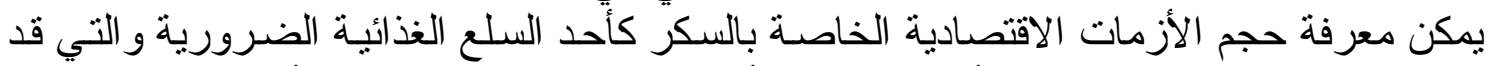

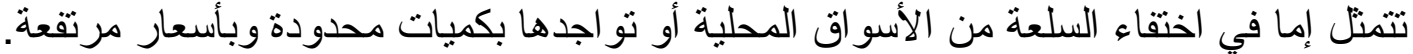

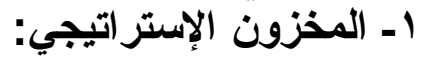

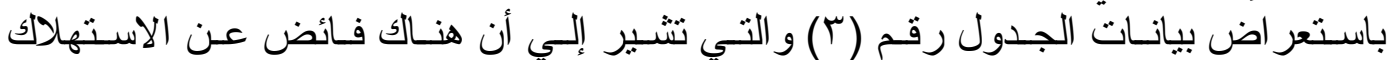

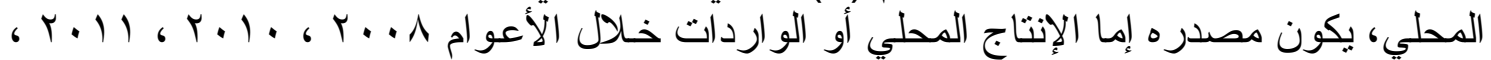

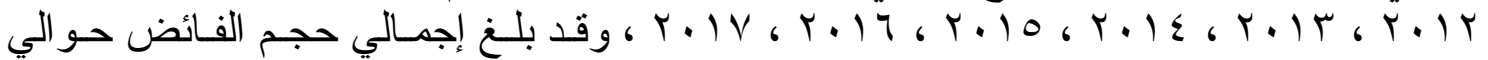

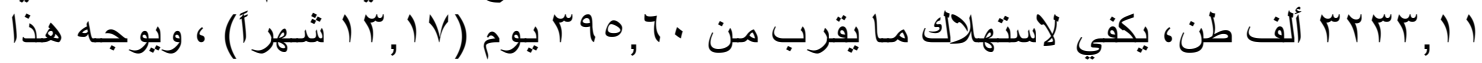

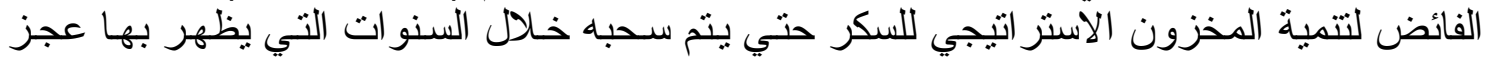
في السكر المتاح للاستهلاك المحلي.

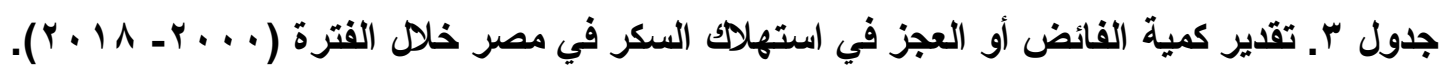

\begin{tabular}{|c|c|c|c|c|c|c|c|c|}
\hline \multicolumn{2}{|c|}{ العجز } & \multicolumn{2}{|c|}{ الفائض } & \multirow[b]{2}{*}{ بالفترثين } & \multirow[b]{2}{*}{ للاسترة تغطية } & \multirow[b]{2}{*}{ لفاسترة كفاية } & \multirow[b]{2}{*}{ الاستهلاك الألفي } & \multirow[b]{2}{*}{ السنة } \\
\hline فلاسترة كفاية & بالألف طن الكمية & لقلاسترة كفاية & بالألف طن & & & & & \\
\hline$I Y, V T$ & $\pi r, 9 r$ & & & TOY, Y & 79,71 & YAY,OT & $\varepsilon, 9 r$ & $r \ldots$ \\
\hline $1, r$ & 0,0 & & & rדז, 9ᄉ & $\vee q, \wedge r$ & $r \wedge \varepsilon, 10$ & $\varepsilon, 90$ & $r \ldots 1$ \\
\hline$r Y, 0 q$ & $I r T, V V$ & & & $r \leq r, \leq 1$ & 91,91 & ro.,o. & $0, \leqslant \wedge$ & $r \cdot r$ \\
\hline$v 9,9 T$ & $£ 09, \wedge \wedge$ & & & rAo, $\cdot V$ & $71,7 V$ & rTr, s. & 0,10 & $r \ldots r$ \\
\hline$\cdot, 7 \varepsilon$ & $r, \wedge \vee$ & & & דוז, & $1 T V, 1 \leq$ & YYV, YY & $7, r$ & $r \cdot . \varepsilon$ \\
\hline$\leqslant 0,0 \leqslant$ & $r \cdot r, \varepsilon \leq$ & & & $19, \leqslant 7$ & $9 \varepsilon, 7 V$ & $r Y \leqslant, V q$ & 7,77 & $r \ldots o$ \\
\hline$\cdot, \mu_{1}$ & $r, 19$ & & & rาะ, 79 & $1 \% 9, \leq 0$ & YYO, Y & 7,99 & $r \ldots r$ \\
\hline$v, 19$ & 01,10 & & & $r \circ V, \wedge I$ & $111, r$ & $r \leqslant 7, \vee q$ & $V, I Y$ & $r \ldots V$ \\
\hline & & $1 \leqslant, r_{0}$ & $1 \cdot r, v 9$ & rvq, ro & $17 ., 01$ & Y\^,VT & V,YT & $r \cdot \Lambda$ \\
\hline 71,14 & $\leqslant 00, \leqslant 0$ & & & $r \cdot r, \wedge \wedge$ & $\Lambda V, V T$ & 417,10 & $V, \leqslant 0$ & $r . .9$ \\
\hline & & $r q, v)$ & $\Gamma \cdot, \wedge l$ & $\varepsilon \cdot \varepsilon, \bigvee)$ & $1 \leqslant 1, \wedge 0$ & rצr,AT & $V, 0 \wedge$ & $r .1$. \\
\hline & & $r \wedge, \vee \uparrow$ & $r q r, 0$ & $\varepsilon \cdot T, \vee T$ & 101,19 & Y01, , 1 & $v, 01$ & $r .11$ \\
\hline & & $r \varepsilon, \wedge r$ & $19 \leqslant, 0 \leqslant$ & 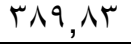 & $1 T \varepsilon, \cdot \varepsilon$ & Yoo, VA & $\vee, \wedge \varepsilon$ & $r .1 r$ \\
\hline & & $r q, V I$ & $r \cdot 1, v 1$ & $\varepsilon \cdot 1, v)$ & 101,70 & $r \leqslant r, 0$ & $\Lambda, Y r$ & $r .1 r$ \\
\hline & & $\Lambda T, O r$ & $T \vee \wedge, Y O$ & $\varepsilon \varepsilon V, O r$ & $17 V, 9$. & $r \vee q, 7 r$ & $\Lambda, Y r$ & $r+1 \varepsilon$ \\
\hline & & $\Lambda \tau, Y \varepsilon$ & $V T r, \varepsilon T$ & $\varepsilon 01, r \varepsilon$ & $1 \vee 1,9$. & rVq, r & $1, \leqslant 9$ & $r+10$ \\
\hline & & $\pi r, \cdot 1$ & ory, , & $\varepsilon Y V, \cdot 1$ & IVT,Y & ror, ,o & 1,77 & $r .17$ \\
\hline & & $1 \cdot, \leqslant V$ & 94,70 & rVo, $\leqslant V$ & $|r|, r \mid$ & ros, 17 & $\wedge, \wedge 0$ & $r \cdot I V$ \\
\hline Yo, TA & 174,9 & $\varepsilon r, 97$ & $r O q, Y \mu$ & $r v \varepsilon, 1 \varepsilon$ & I YO, YO & $r \leqslant \wedge, \wedge q$ & $v, 11$ & المتوسط \\
\hline
\end{tabular}

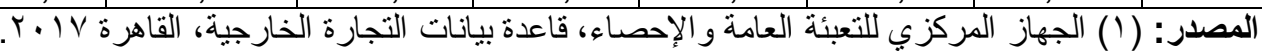

(Y) وزارة الزر اعة و استصلاح الأر اضي، مجلس المحاصيل السكرية، نشرة التقرير السنوي، أعداد متفرقة. 


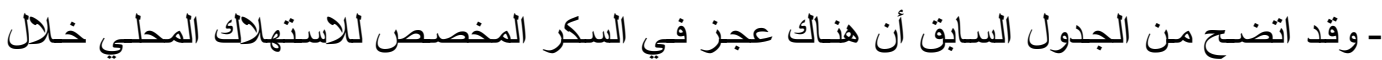

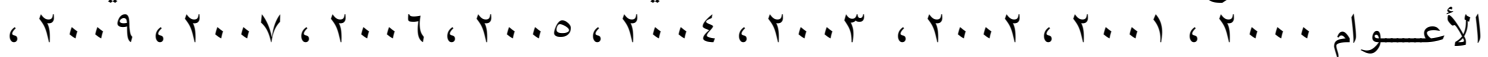

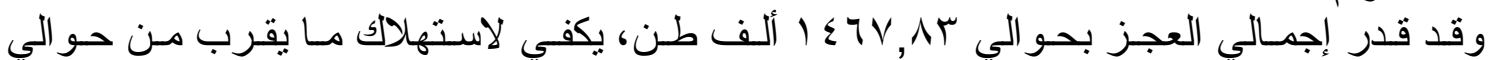

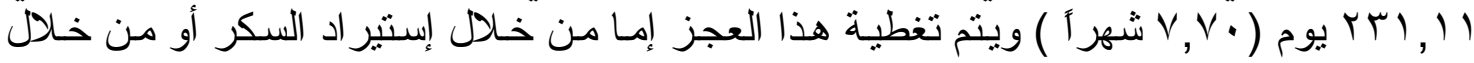
السحب من المخزون الاستر اتيجي.

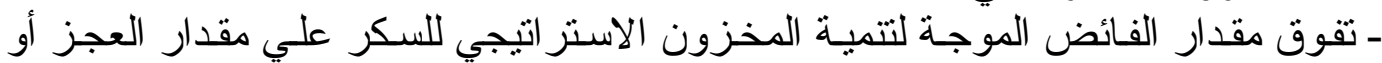

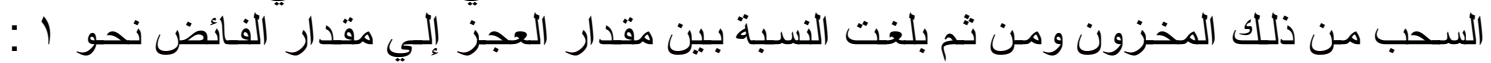

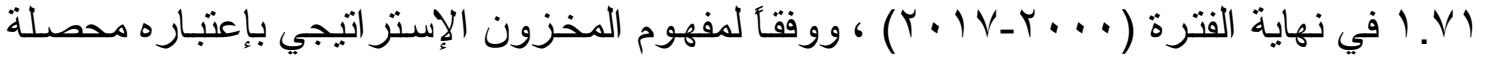

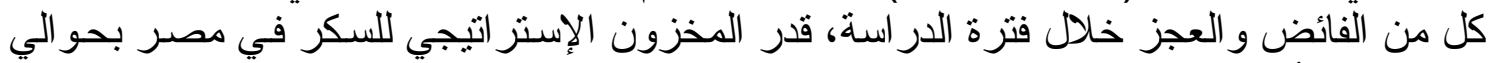

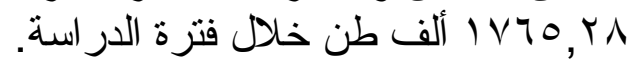

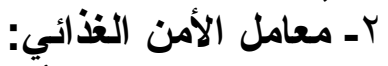

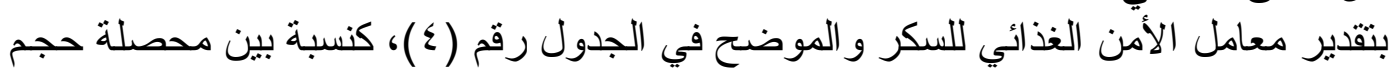

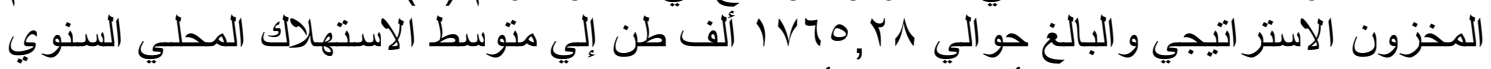

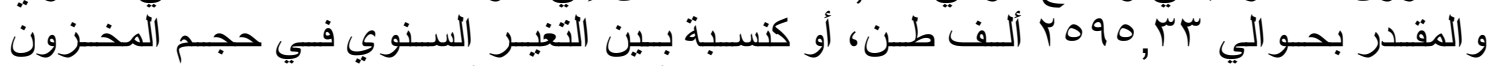

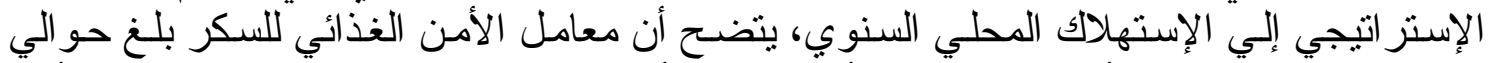

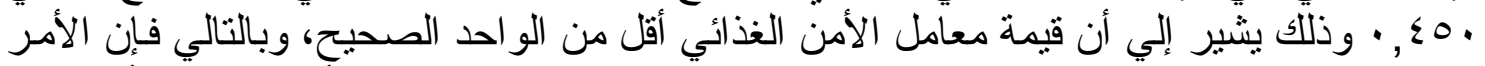

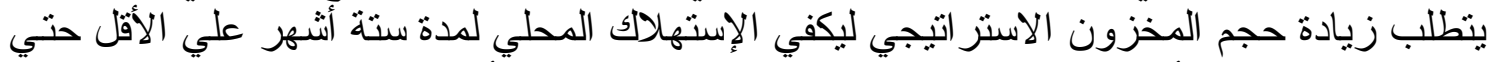

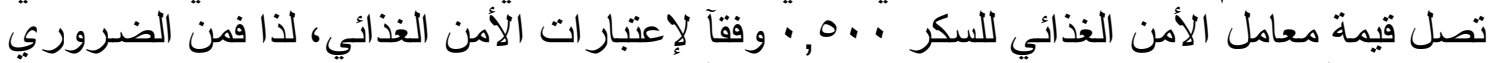

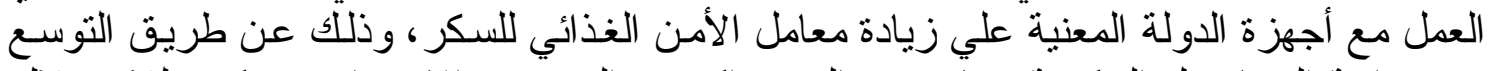

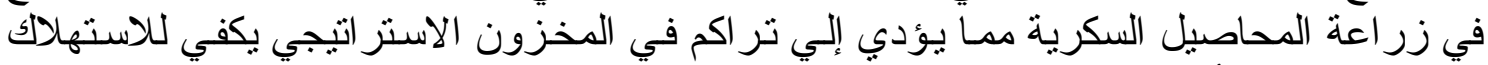

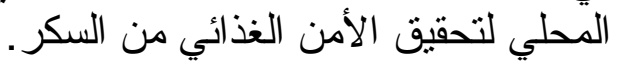

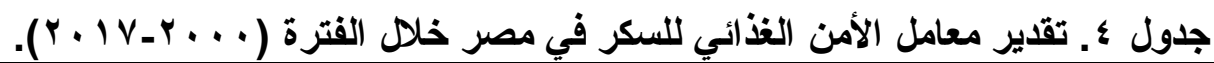

\begin{tabular}{|c|c|c|}
\hline نسبة التغير ف المخزون الاستر اتيجي الي الاستهلاك المحلي & مقار التغير ف المخزون الآستر اتيجي ألَف طن ذن & السنوات \\
\hline$\cdot, \cdot \theta_{-}$ & Tr,94- & $r \ldots$ \\
\hline$\cdot, \cdots r$ & $0, \cdot 0_{-}$ & $r \ldots 1$ \\
\hline$\cdot, \cdot T Y_{-}$ & ITr,V & $r \ldots r$ \\
\hline$\cdot, Y \backslash 9$. & $\varepsilon 09, \wedge \Lambda$ & $r \ldots r$ \\
\hline$\cdot, \cdots Y_{-}$ & $r, \wedge V_{-}$ & $Y \ldots \varepsilon$ \\
\hline$\cdot, 1 Y O_{-}$ & $r \cdot r, \varepsilon \leqslant-$ & $r \ldots o$ \\
\hline$\cdot, \cdots 1-$ & $r, 19-$ & $r \ldots r$ \\
\hline$\cdot, \cdot r \cdot-$ & $01, Y O_{-}$ & $r \ldots v$ \\
\hline$\cdot, \cdot r q$ & $1 \cdot r, v q$ & $r \ldots \Lambda$ \\
\hline$\cdot, 17 V_{-}$ & $\leqslant 00, \leqslant 0_{-}$ & $r \ldots q$ \\
\hline$\cdot, 1 \cdot 9$ & $r \cdots, \wedge !$ & $r+1$. \\
\hline$\cdot, 1 \cdot 7$ & rqY,.0 & $r \cdot 11$ \\
\hline$\cdot, \cdot 7 \Lambda$ & $19 \leqslant, 0 \leqslant$ & $r \cdot I r$ \\
\hline$\cdot, 1 \cdot 1$ & $r \cdot 1, v 1$ & $r \cdot 1 r$ \\
\hline$\cdot, Y Y T$ & $T \vee \Lambda, Y_{0}$ & $r+1 \leq$ \\
\hline$\cdot$, YTT & $V T r, \varepsilon r$ & $r+10$ \\
\hline$\cdot, 1 \mathrm{r}$ & ory,A1 & $Y .19$ \\
\hline$\cdot, \cdot 4 q$ & $9 Y, 70$ & $r \cdot I V$ \\
\hline$\cdot, \leqslant 0$ & IVTQ,YA & الإجمالي \\
\hline
\end{tabular}

المصدر: جمعت وحسبت من: بيانات جدول رقم (r). 
ثالثاً: العوامل الإقتصادية المحددة لمعامل الأمن الغذائي في مصر.

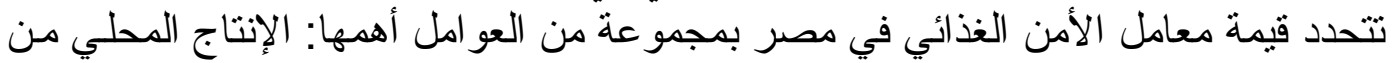

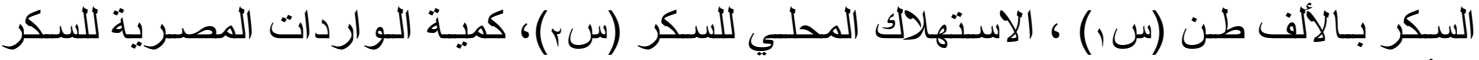
بالألف طن (سب).

وبإجر اء تحليل الانحدار المتعدد للمتغير ات الاقتصادية المحددة لمعامل الأمن الغذائي للسكر

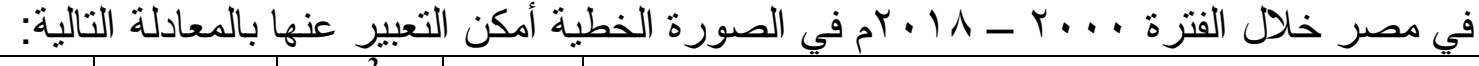

\begin{tabular}{|c|c|c|c|c|}
\hline D.W & $\begin{array}{c}\text { F } \\
\text { "المسوبة } \\
\text { "الفوبة }\end{array}$ & $\begin{array}{l}\mathbf{R}^{2} \\
\text { معلديد } \\
\end{array}$ & $\begin{array}{c}\mathbf{R} \\
\text { الارتباطط } \\
\end{array}$ & المعادلة \\
\hline 1,710 & $* * \mu V \Psi, Y$ & $\cdot, 9 \wedge \wedge$ & $\cdot, 99 \leqslant$ & 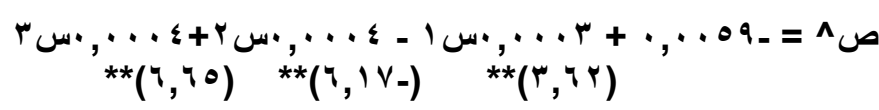 \\
\hline
\end{tabular}

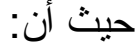

$$
\begin{aligned}
& \text { ص =معامل الأمن الغذائي } \\
& \text { س, =إنتاج السكر المحلي ألف طن طن } \\
& \text { سب =الإستهالاك المحلي ألف طن طن } \\
& \text { سبr =كمبة الواردات ألف طن } \\
& \text { D.W اختيار دربن و اتسن }
\end{aligned}
$$

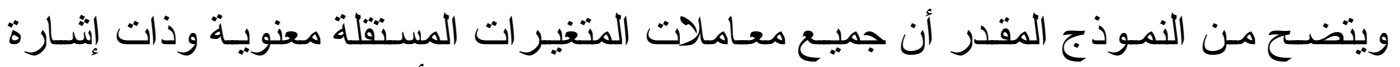

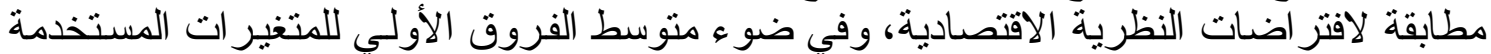

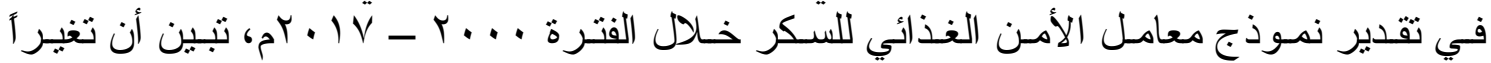

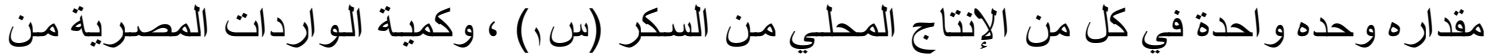

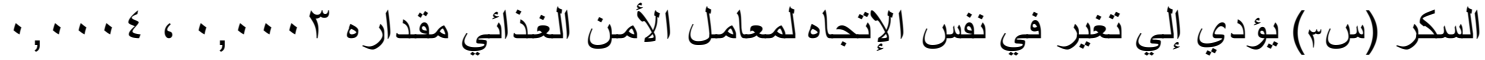

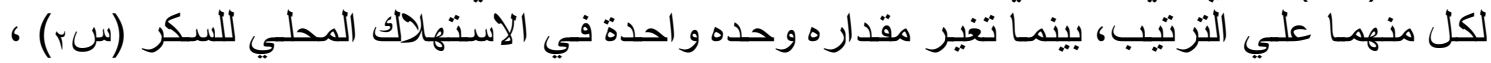

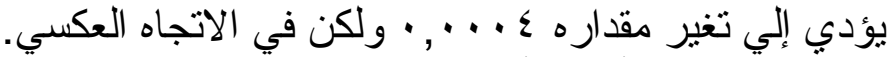

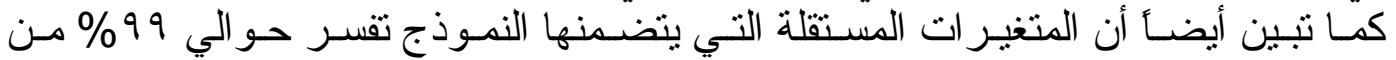

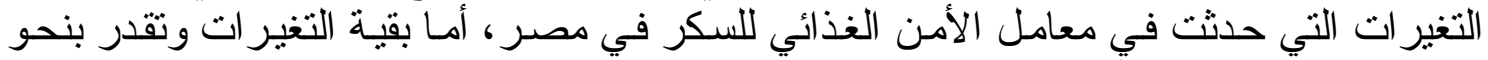

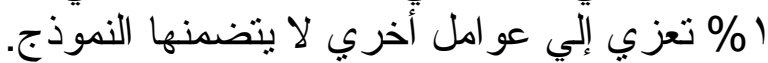

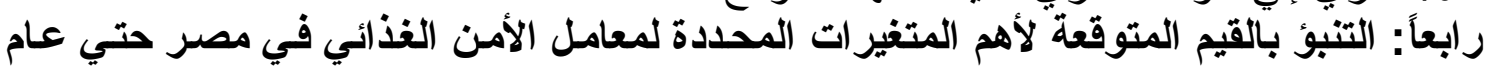
r.r.

في ضو و الاستر اتيجية المستقبلية للزر اعة المصرية، وبإستعر اض بيانات الجدول رقم (0)

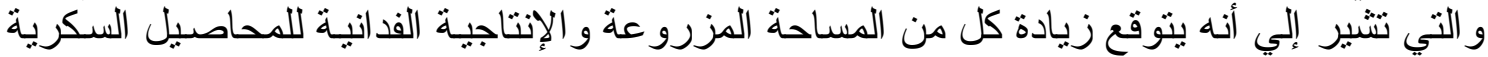

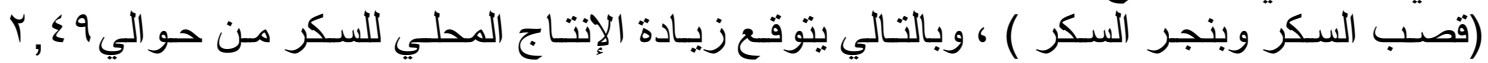

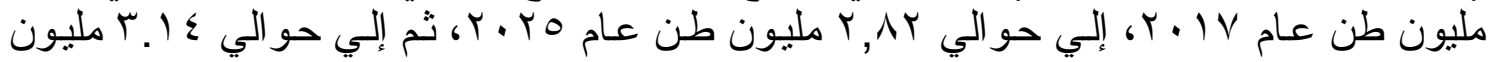

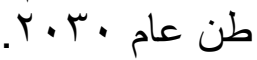

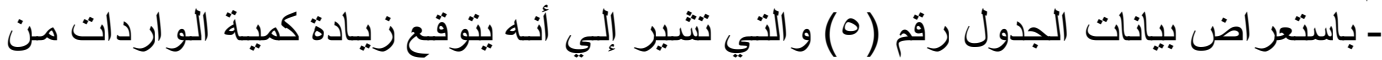

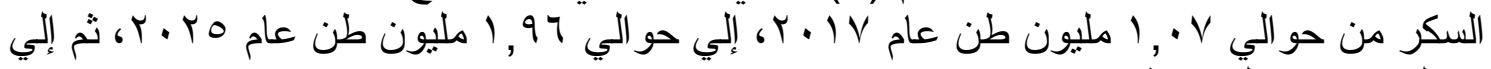

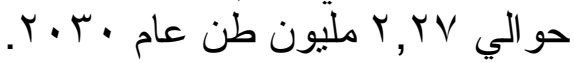

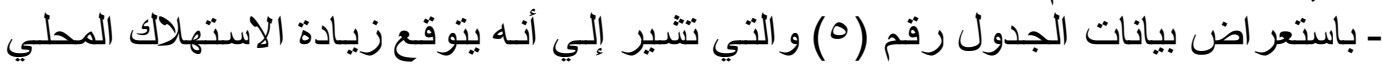

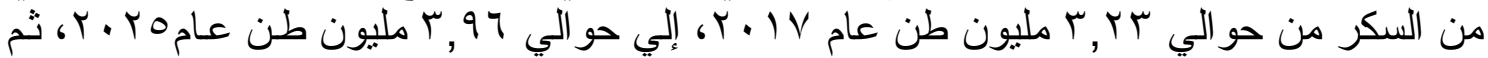

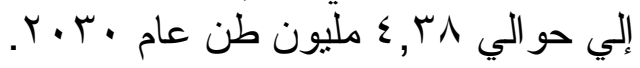


جدول ه. التبؤ بـالقيم المتوقعة لأهم المتغيرات المحددة لمعامـل الأمـن الغذائي في مصر حتـي عـام

r.r.

\begin{tabular}{|c|c|c|c|}
\hline pr.r. & br. ro & $5 r+1 V$ & البيان \\
\hline & & & المساحة بالألف فان: \\
\hline rrT, & $r r \cdot, 9 T$ & 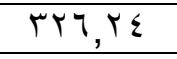 & قصب السكر \\
\hline \multirow[t]{2}{*}{$940, \leqslant 9$} & $V q \cdot, r Y$ & OrT,rN & بنجر السكر \\
\hline & & & الإنتاجية الفدانية بالطن: \\
\hline$\leqslant 0,0 Y$ & $\varepsilon 7, r V$ & $\leqslant V, 10$ & قصب السكر \\
\hline \multirow[t]{2}{*}{$Y, 01$} & YI, $\varepsilon$ & $r \cdot, V_{0}$ & بنجر السكر \\
\hline & & & الإنتاج المحلي للمحاصيل السكرية بالألف طن: \\
\hline 10Y9Y,r & $10 \leqslant \leqslant V, 7$ & lOKAT,YI & قصب السكر \\
\hline $19 \vee 7 \varepsilon, 9$ & $177 \wedge \varepsilon, r$ & $1 \cdot 17 \cdot, 9 r$ & بنجر السكر \\
\hline TI $\leqslant V, O V$ & YAYV,IA & $r Y \leqslant 9,10$ & الإنتاج المحلي للسكر للإجمالي بالألف طن \\
\hline KrVq, $\leqslant V$ & $197 \leq, 14$ & $1 \cdot V r, 0 \cdot$ & كمية الواردات بالألف طن \\
\hline 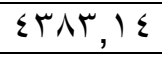 & rqTV,rV & rrm.,. & الكمية المستهلكة بالألف طن \\
\hline$\varepsilon \cdot, r$ & $r \Lambda, 00$ & $r \varepsilon, \cdots$ & متوسط نصيب الفرد بالكيلو جرام \\
\hline$\vee 1, \vee q$ & $V \cdot, 97$ & 79,74 & نسبة الاكتفاء الأاتي للسكر (\%) \\
\hline $14, \cdot 1$ & $1 \cdot, \wedge \mathrm{V}$ & $\wedge, \wedge 0$ & الاستهلاك المحلي اليومي للسكر بالألف طن \\
\hline YTY, $: \varepsilon$ & ro^, $9 \wedge$ & ros,17 & فترة كفاية الإنتاج للاستهلاك المحلي باليوم \\
\hline IYYA, rV & 1110,77 & $9 \wedge 1, \cdots$ & حجم الفجوة من السكر بالألف طن \\
\hline
\end{tabular}

المصدر: جمعت وحسبت من: بيانات الجدول رقم (ז).

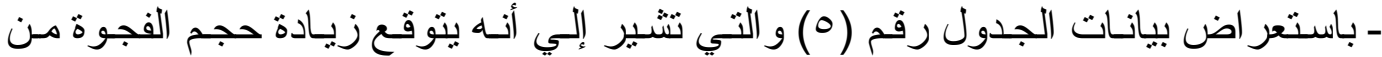

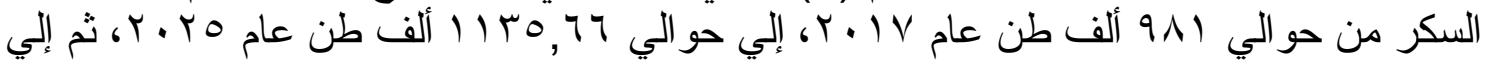

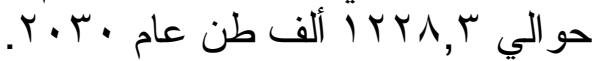

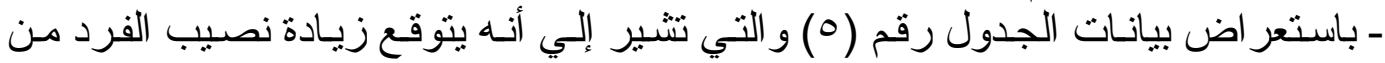

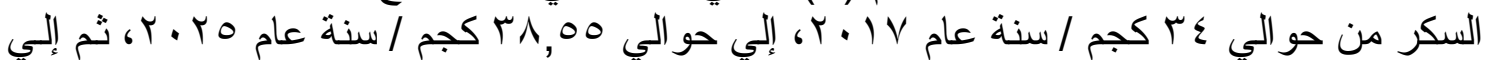

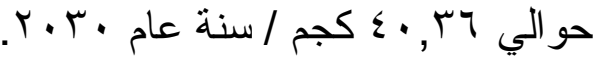

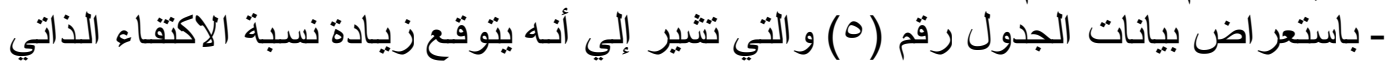

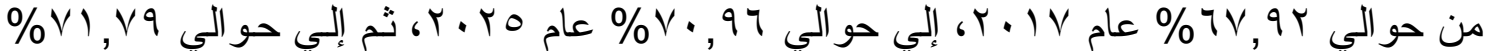

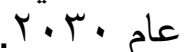
- باستعر اض بيانات الجدول رقم (0) حيث تشير إلي أنسه يتوقع زيـادة الاستهلاك المحلي

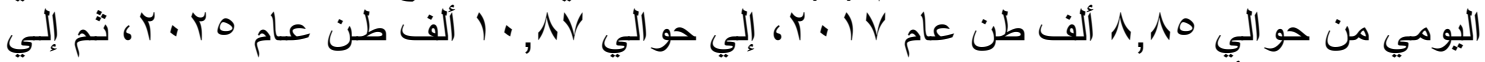

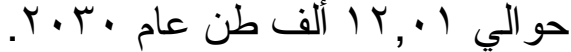

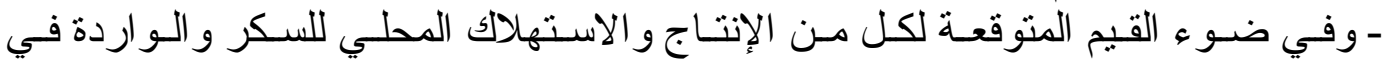

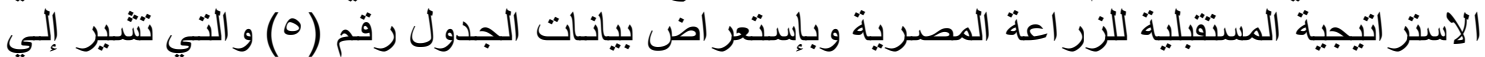

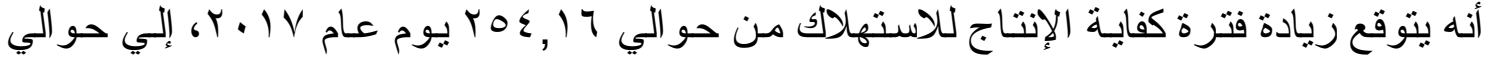

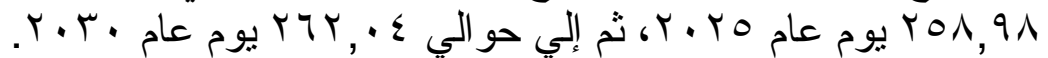

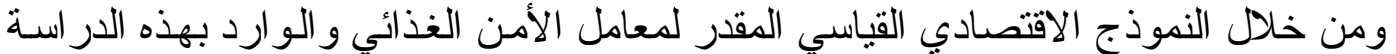

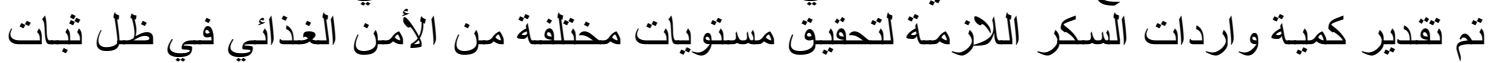

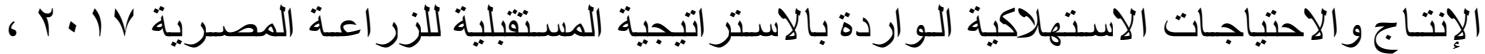




\section{باستعر اض بيانات الجدول رقم (T) يتضح أنها تنشير إلي مايلي:}

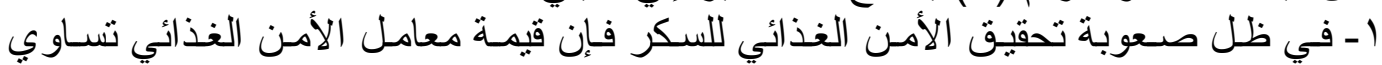

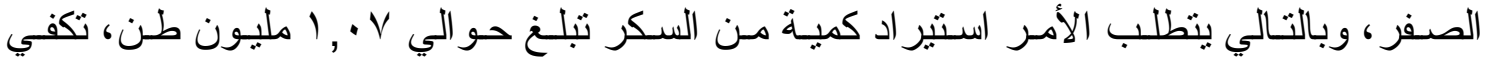

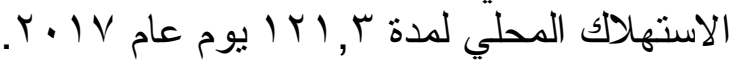

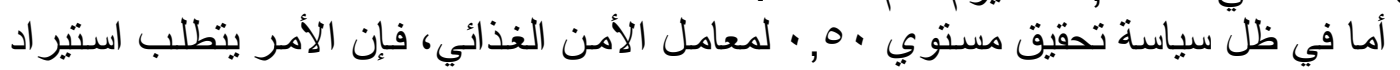

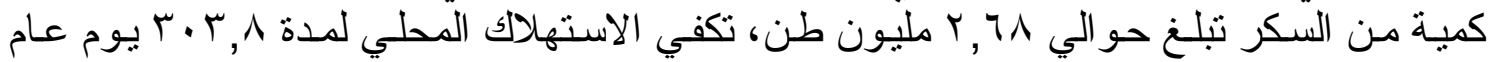

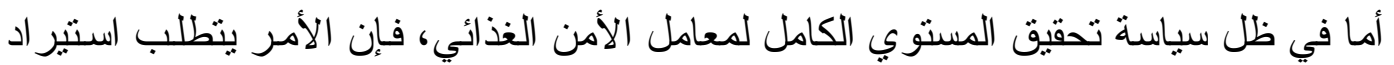

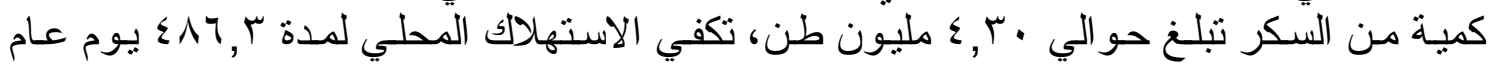
$r \cdot 1 Y$

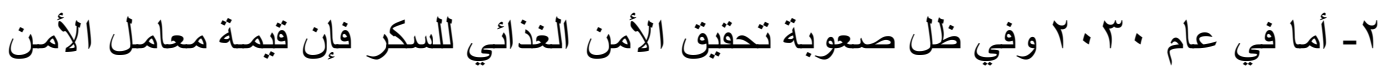

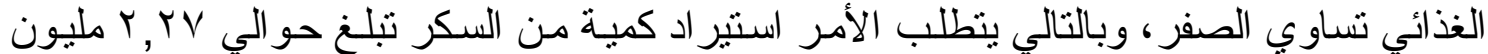

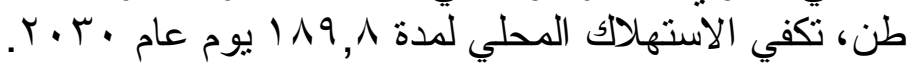

جدول 7. $r \cdot r \cdot$. r. IV

\begin{tabular}{|c|c|c|c|c|}
\hline \multicolumn{2}{|l|}{$r \cdot r}$. & \multicolumn{2}{|l|}{$r+I V$} & \multirow[b]{2}{*}{ معامل الأمن الغذائي } \\
\hline 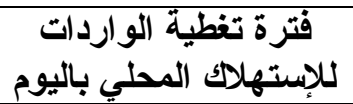 & كمية الواردات بات & للإسترهلاك المحلة الوارداتي باليوم & كمية الواردات & \\
\hline $1 \wedge 9, \wedge$ & KYVQ,O & $|r|, r$ & $1 \cdot V r, 0$ & $\cdot$, \\
\hline TYY, & TVIV,A & $10 \mathrm{~V}, 1$ & 1199,0 & .1 \\
\hline TTY,A & $\Gamma 107, r$ & $19 \varepsilon, r$ & 1819,7 & $\cdot, r$ \\
\hline rqq,r & roq, 7 & $r M \cdot, \Lambda$ & $r \cdot \leqslant Y, T$ & $\cdot, r$ \\
\hline & $\varepsilon \cdot r Y, q$ & YTV,r & TrTo,T & $\cdot, \varepsilon$ \\
\hline MVY,T & $\varepsilon \varepsilon V \backslash, r$ & $r \cdot r, \wedge$ & 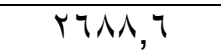 & $\cdot, 0$ \\
\hline$\varepsilon \cdot \wedge, \wedge$ & $\varepsilon 9.9, v$ & $\Gamma \varepsilon \cdot, r$ & $r \cdot 11, v$ & $\cdot, 7$ \\
\hline$\leqslant \leqslant 0, r$ & $0 r \leqslant \Lambda$, & $r \vee \tau, \wedge$ & TrT & $\cdot, \mathrm{V}$ \\
\hline$\varepsilon \wedge 1, \wedge$ & OVAT, \& & $\varepsilon \|, r$ & rev, , V & $\cdot, \wedge$ \\
\hline $01 \wedge, r$ & $T Y Y \leqslant, \Lambda$ & $\varepsilon \leqslant 9, \wedge$ & $r 9 \Lambda \cdot, v$ & $\cdot, 9$ \\
\hline $00 \leqslant, 1$ & 7774,1 & $\varepsilon \wedge \neg, \Gamma$ & $\varepsilon r \cdot r, \Lambda$ & 1, \\
\hline
\end{tabular}

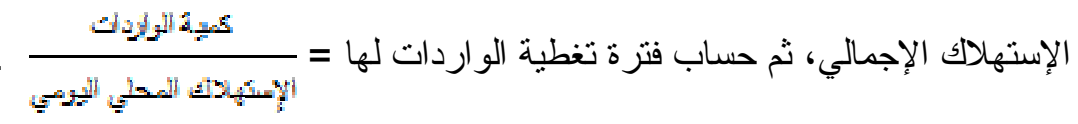

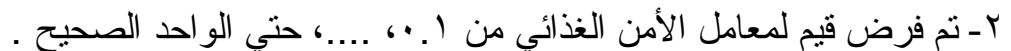

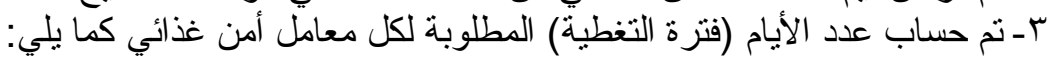

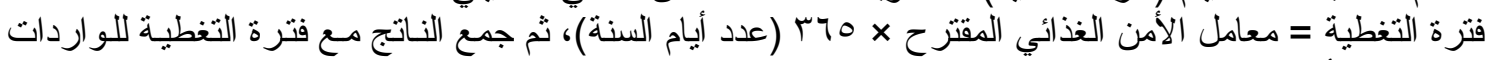

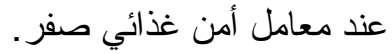

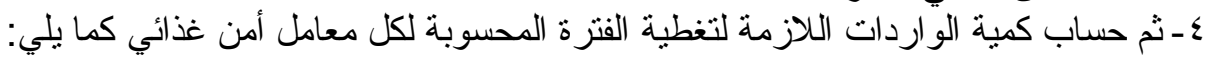

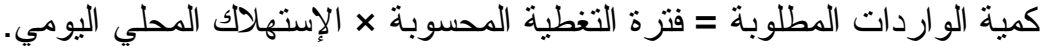

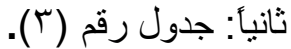

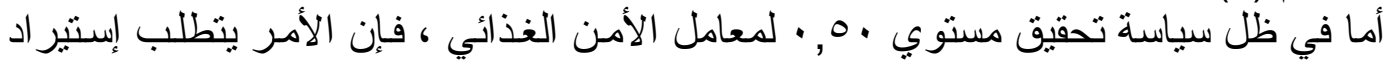

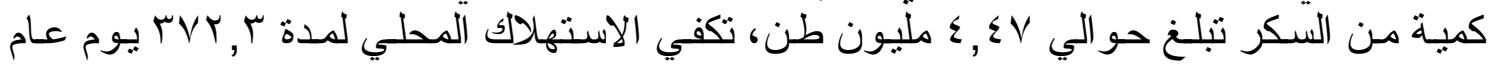




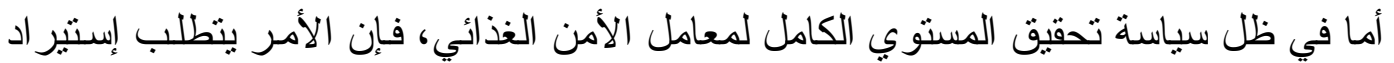

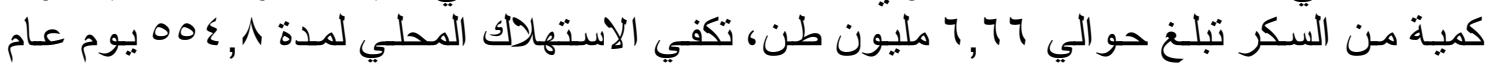
r.r. الملخص

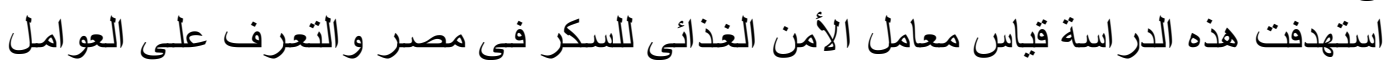

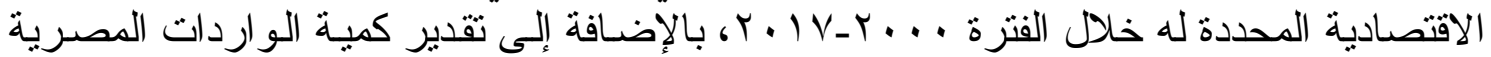

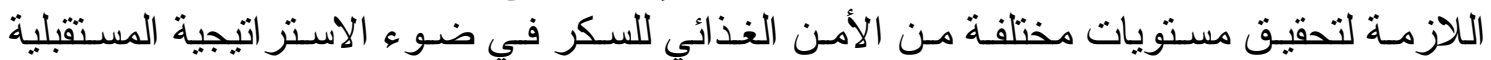

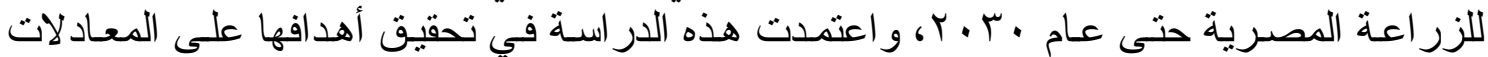

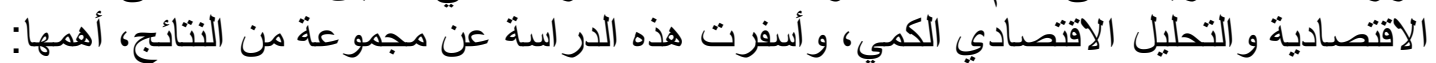

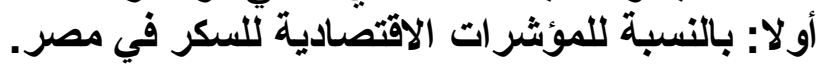

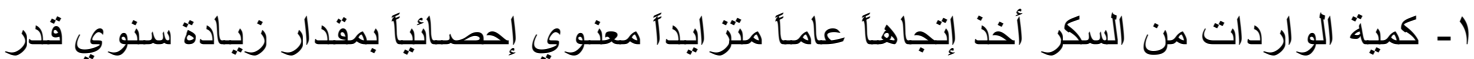

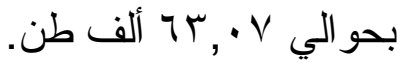
r- الكمية المستهلكة من السكر أخذت إتجاهاً عامـأ متز ايدأ معنوي إحصـائياً بمقدار زيادة سنوي

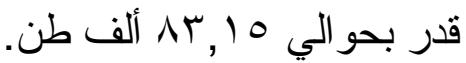
r- حجم الفجوة من السكر أخذت إتجاهاً عامـاً متز ايدأ معنوي إحصـائياً بمقدار زيادة سنوي قدر

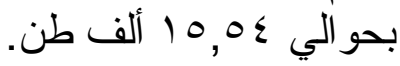
عـ متوسط نصيب الفرد من السكر أخذ إتجاها عاماً متز ايداً معنوي إحصائياً بمقدار زيادة سنوي

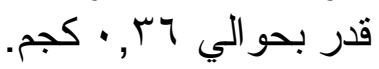

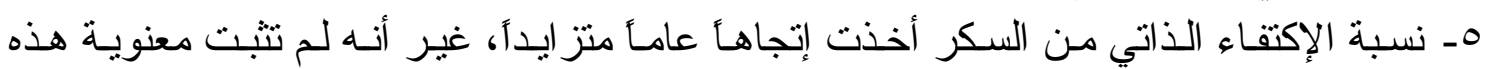
الزيادة.

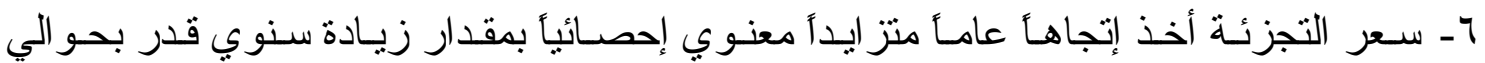

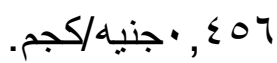
V- سعر السكر عالمياً أخذ إتجاهاً عاماً متز ايداً، غير أنه لم تثتبت معنوية هذه الزيادة.

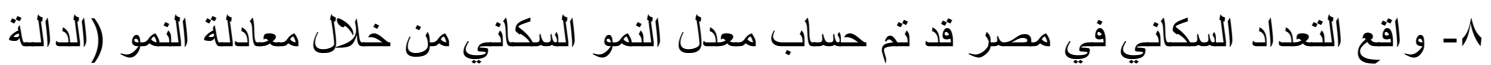

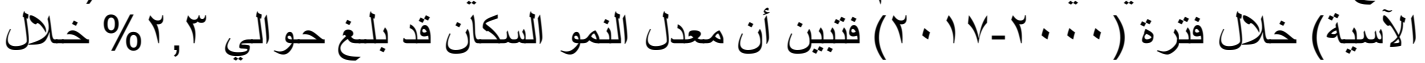

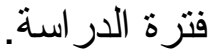
ثانياً: بالنسبة لمعامل الأمن الغذائي:

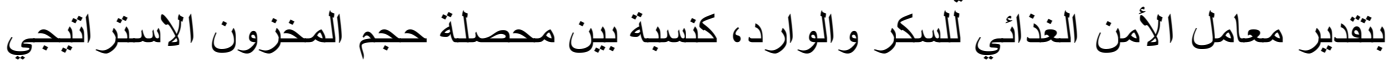

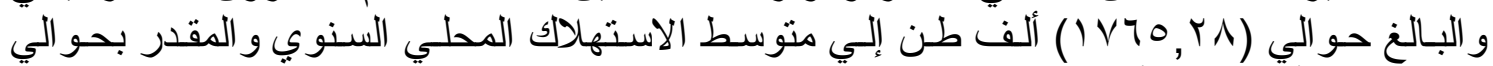

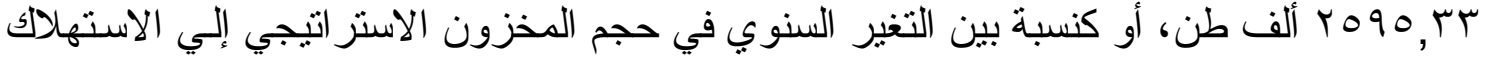

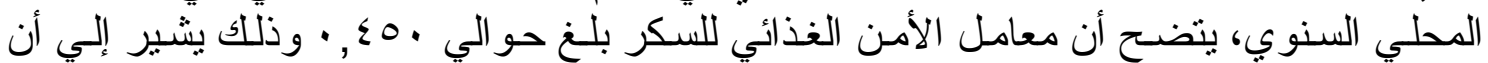

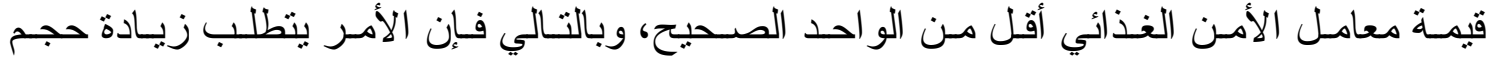

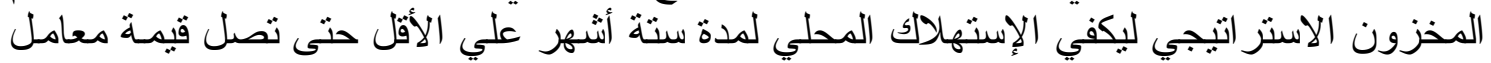

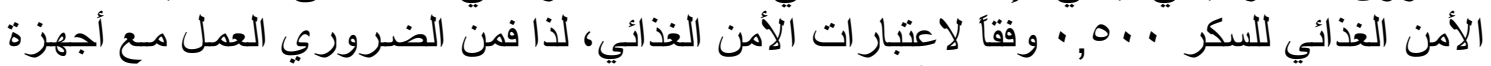

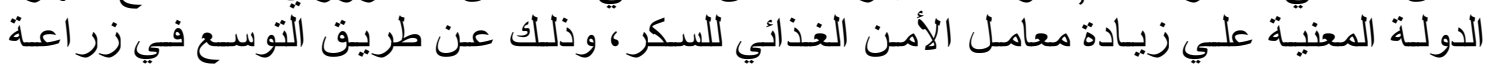

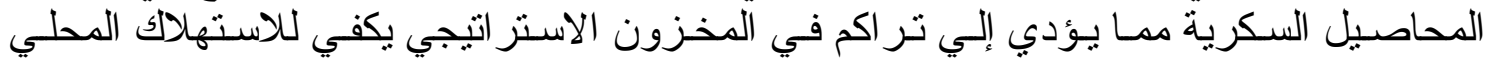

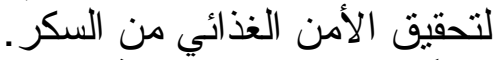

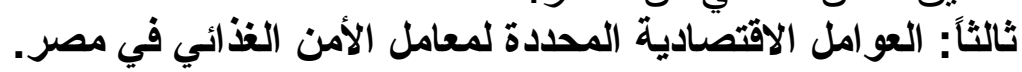

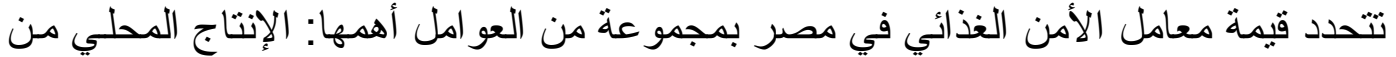

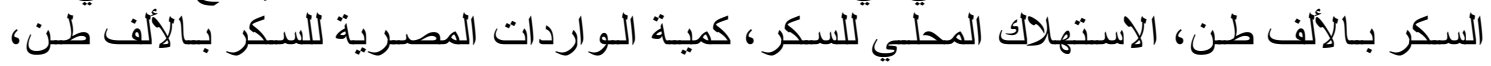




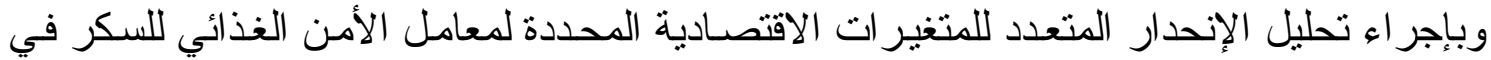

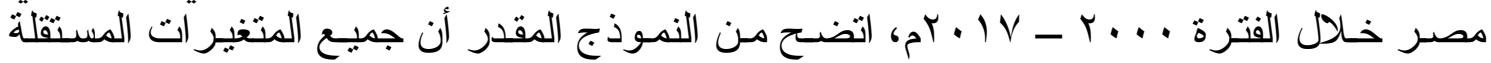
بدرجة معنوية وذات إثنارة مطابقة لافتز اضات النظرية الاقتصنادية. التوصيات:

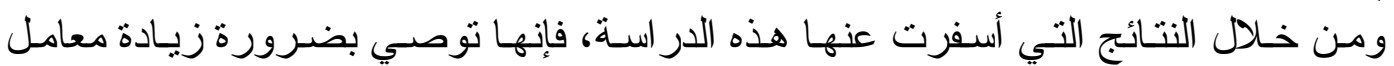

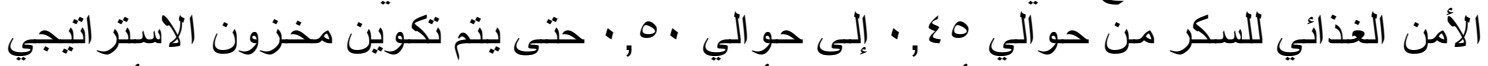

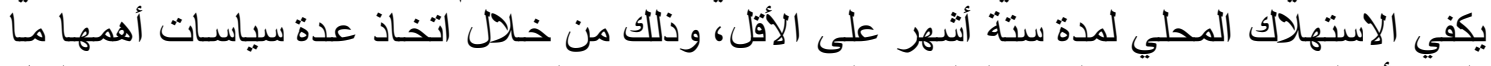

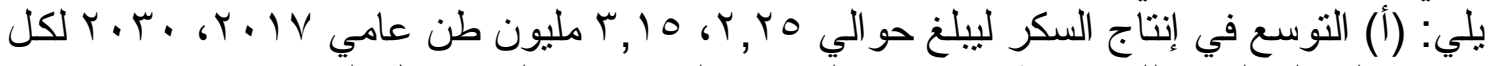

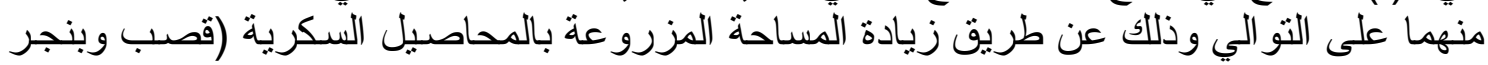

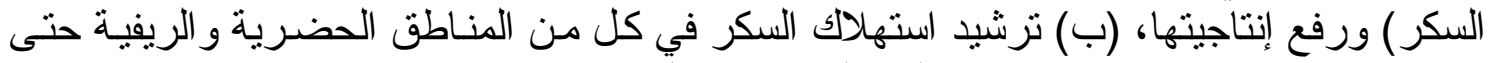

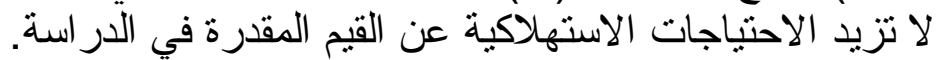

الجهاز المركزي للتعبئـة العامــة والإحصـاء، نشـرة اسـتهلاك الســـع في جمهوريـة مصـر العربيـة، أعداد

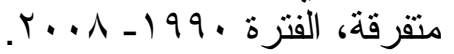

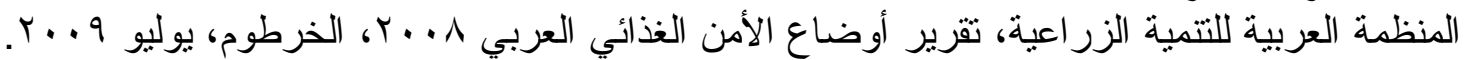

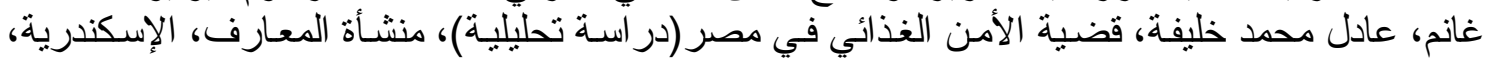
$.199 \mathrm{~V}$

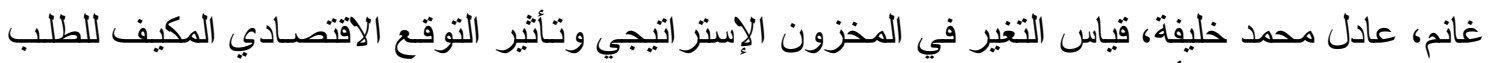

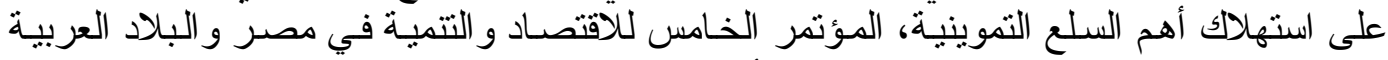

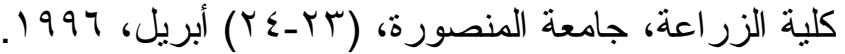

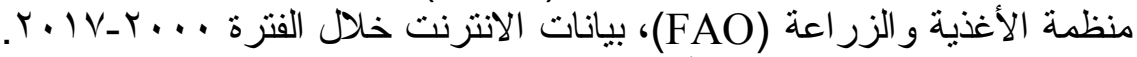

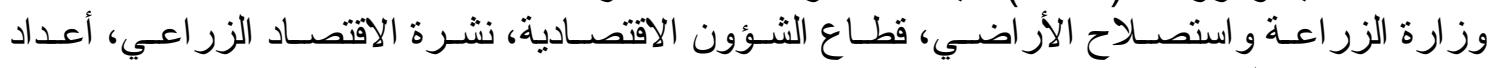

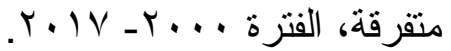

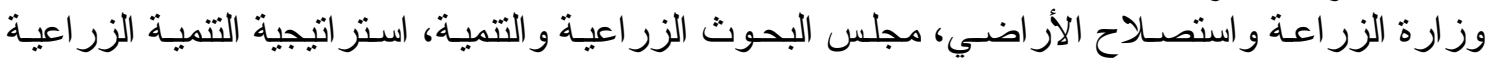

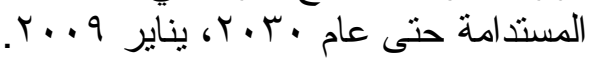

Dickey, D. and Fuller, W. Distribution of the estimators for auto-regressive time series with a unit root. Journal of the American Statistical Association, Vol. 74, 1979, 427-431.

William H. Greene, Econometric Analysis, Fifth edition, New York University, 2003. 


\title{
A Study of the Specific Economic Factors for Suger Food Security Laboratores in Egypt
}

\section{Alla G. Esmail; M.A. Abonahoul; Atif H. EIShemy and Talat H. Esmail}

\author{
Agricultural Economics- Faculty of Agriculture - Assiut University
}

\section{Summary}

This study aimed to measure the food security coefficient of sugar in Egypt and identify the specific economic factors for it during the period 2000-2017, in addition to estimating the amount of Egyptian imports needed to achieve different levels of food security for sugar in light of the future strategy of Egyptian agriculture until 2030. This study relied, in achieving its goals, on economic equations and quantitative economic analysis. This study yielded a set of results, the most important of which are:

First: With regard to the economic indicators of sugar in Egypt.

1- The quantity of imports of sugar has taken an increasing trend, statistically significant, with an annual increase estimated at about 63.07 thousand tons.

2- The amount of sugar consumed took an increasing trend, statistically significant, with an annual increase estimated at about 83.15 thousand tons.

3- The size of the sugar gap has taken an increasing general statistically significant trend, with an annual increase estimated at 15.54 thousand tons.

4- The average per capita sugar takes an increasing general trend, statistically significant, with an annual increase estimated at $0.36 \mathrm{~kg}$.

5- The rate of self-sufficiency in sugar took an increasing general trend, but it did not prove the significance of this increase.

6- The retail price took a general, statistically significant trend, with an annual increase estimated at about 0.445 pounds $/ \mathrm{kg}$.

7- The price of sugar globally is taking an increasing general trend, but it did not prove the significance of this increase.

8- The reality of the population census in Egypt, the population growth rate was calculated through the growth equation (exponential function) during the period (2000-2017), so it was found that the population growth rate reached $2.3 \%$ during the study period).

Second: With regard to food security laboratories:

By estimating the food security factor for sugar and incoming, as a ratio between the sum of the strategic stock size of about $(1765,28)$ thousand tons to the average annual domestic consumption estimated at 2595.33 thousand tons, or as a ratio between the annual change in the size of the strategic stock to the annual domestic consumption, it becomes clear that the coefficient of Food security for sugar amounted to about 0.450 and this indicates that the value of the food security coefficient is less than the correct one, and therefore it is necessary to increase the size of the strategic stockpile so that domestic consumption suffices for at least six months until the value of the food security coefficient of sugar reaches 0,500 according to food security considerations so it is $I t$ is necessary to work with the concerned state agencies to increase the food security factor for sugar, by expanding the cultivation of sugar crops, which leads to an 
accumulation in the strategic stockpile sufficient for local consumption to achieve food security from sugar.

Third: The specific economic factors for food security laboratories in Egypt:

The value of the food security coefficient in Egypt is determined by a set of factors, the most important of which are: local production of sugar per thousand tons, local consumption of sugar, the amount of Egyptian imports of sugar per thousand tons, and by conducting a multiple regression analysis of the economic variables identified for the food security coefficient of sugar in Egypt during the period 2000 - $2018 \mathrm{AD}$, and it became clear From the estimated model, all the independent variables are significantly significant and have a reference identical to the assumptions of economic theory. 